\title{
Optimal Information Disclosure in Auctions and the Handicap Auction*
}

\author{
Péter Esô \\ Northwestern University, Kellogg School, MEDS \\ Balázs Szentes ${ }^{\ddagger}$ \\ University of Chicago, Department of Economics
}

November 2007

\begin{abstract}
We analyze a situation where a monopolist is selling an indivisible good to risk neutral buyers who only have an estimate of their private valuations. The seller can release, without observing, certain additional signals that affect the buyers' valuations. Our main result is that in the expected revenue maximizing mechanism, the seller makes available all the information that she can, and her expected revenue is the same as it would be if she could observe the part of the information that is "new" to the buyers.

We also show that this mechanism can be implemented by what we call a handicap auction in interesting applications. In the first round of this auction, each buyer picks a price premium from a menu offered by the seller (a smaller premium costs more). Then the seller releases the additional signals. In the second round, the buyers bid in a second-price auction where the winner pays the sum of his premium and the second highest non-negative bid. In the case of a single buyer, this mechanism simplifies to a menu of European call options.
\end{abstract}

JEL Classification: C72, D44, D82, D83

${ }^{*}$ Revised version of our article in the Review of Economic Studies, Volume 74 (2007), pp. 705-731. We thank Mark Armstrong, Yeon-Koo Che, Kim-Sau Chung, Eddie Dekel, Drew Fudenberg, Peter Klibanoff, Marco Ottaviani, Alessandro Pavan, Andrew Postlewaite, Philip Reny, James Schummer, Nancy Stokey, Lucy White, seminar participants at Caltech, Chicago, Harvard-MIT, LBS, LSE, Northwestern, Princeton, Southampton, Toronto, UCL, UPenn, Virginia Polytechnic, Wisconsin-Madison, the 2002 Midwest Theory Meeting, and the 2003 Summer Meeting of the Econometric Society for comments, and Norbert Maier for research assistance. We are grateful to the Editor and two referees for their suggestions. Szentes thanks the NSF for financial support.

${ }^{\dagger} 2001$ Sheridan Rd, Evanston, IL 60208. Email: eso@northwestern.edu.

$\ddagger 1126$ E. 59th St, Chicago, IL 60637. Email: szentes@uchicago.edu. 


\section{Introduction}

In many examples of the monopolist's selling problem (optimal auctions), ${ }^{1}$ the seller has considerable control over the accuracy of the buyers' information concerning their own valuations. Often the seller can decide whether the buyers can access information that refines their valuations; however, she either cannot observe these signals, or at least, she is unaware of their significance to the buyers. For example, the seller of an oil field or a painting can determine the number and nature of the tests the buyers can carry out privately (without the seller observing the results). Another example (due to Bergemann and Pesendorfer (2002)) is where the seller of a company has detailed information regarding the company's assets (e.g., its client list), but does not know how well these assets complement the assets of the potential buyers. Here, the seller can choose the extent to which she will disclose information about the firm's assets to the buyers. In other applications (e.g., selling broadcast rights for a future sports event) the buyers' valuations for the good become naturally more precise over time as the uncertainty resolves, and the seller can decide how long to wait with the sale.

When the buyers' information acquisition is controlled by the seller, that process can also be optimized by the mechanism designer. In the present paper we explore the revenue maximizing mechanism for the sale of an indivisible good in a model where the buyers initially only have an estimate of their private valuations. The seller can costlessly release additional private signals to the buyers that affect their valuations. These signals may be correlated with the buyers' private information, they are not observable to the seller, and the seller decides whether a buyer can observe them. This model captures the common theme of the motivating examples: the seller controls, but cannot learn, certain private information that the buyers care about. ${ }^{2}$

Our main result is that in the optimal mechanism (and under certain conditions) the seller releases all the information she can, and her expected revenue is as high as it would be if she could observe the part of the information controlled by her that is "new" to the buyers. That is, in the optimal mechanism, the buyers do not enjoy additional

\footnotetext{
${ }^{1}$ Early seminal contributions include Myerson (1981), Harris and Raviv (1981), Riley and Samuelson (1981), and Maskin and Riley (1984).

${ }^{2}$ Note that in some of the motivating examples the seller did observe the "message" disclosed to the potential buyer, but importantly, she did not observe its effect on the buyer's valuation. This situation is modeled by the assumption that the seller can release, without observing, private signals to the buyers.
} 
informational rents from learning more about their ex-post valuations when the access to additional signals is controlled by the seller.

We emphasize that the information disclosure policy of the seller does not have to be a zero-one decision. That is, we could allow the seller to reveal her information only partially. For example, she could show an element of a partition where the new information belongs to, or reveal a signal that is only imperfectly correlated to the new information. Our result still continues to hold: In the optimal mechanism the seller reveals all her information.

We also exhibit a simple mechanism, dubbed the handicap auction, which implements the revenue-maximizing outcome in some interesting applications of the general model. The first application is one where the buyers' original information pertains solely to the expected value of their valuation. (That is, each buyer's true valuation differs from his initial estimate by an independent noise.) In the second application each buyer's ex-post valuation is the realization of a normally distributed random variable. The buyers' initial estimates and the seller's signals are normally distributed, conditionally independent noisy observations of the buyers' true valuations. Note that in this "sampling" application a buyer's private information and the signal controlled by the seller are strictly affiliated. ${ }^{3}$

The handicap auction, which implements the optimal mechanism in the two applications above, consists of two rounds. In the first round, each buyer buys a price premium from a menu provided by the seller (a smaller premium costs more). Then, without observing, the seller releases as much information as she can. In the second round, the buyers bid in a second-price auction, where the winner is required to pay his premium over the second highest non-negative bid. We call the whole mechanism a handicap auction because buyers compete under unequal conditions in the second round, where a bidder with a smaller premium has an advantage.

When there is only a single buyer, the handicap auction simplifies to a mechanism that can be thought of as a menu of European call options offered by the seller. These types of options are widely used in financial forward markets. In our model, as in reality, an option with a higher strike price costs more to the buyer. Buyers with different initial estimates regarding the good's value sort themselves and choose different options.

Our model nests the classical (independent private values) auction design problem

\footnotetext{
${ }^{3}$ We thank Marco Ottaviani for suggesting that we develop an application along these lines.
} 
as a special case, where the additional signals are identically zero. In this case, the handicap auction implements the outcome of the optimal auction of Myerson (1981) and Riley and Samuelson (1981).

Information disclosure by the seller in an auction has been studied in the context of the winner's curse and the linkage principle by Milgrom and Weber (1982). They investigate whether in traditional auctions the seller should commit to disclose public signals that are affiliated with the buyers' valuations. They find that the seller gains from committing to full disclosure, because that reduces the buyers' fear of overbidding, thereby increasing their bids and hence the seller's revenue. Our problem differs from this classic one in many aspects. Most importantly, in our setting, the signals that the seller can release are private (not public) signals, in the sense that each signal affects the valuation of a single buyer and can be disclosed to that buyer only. The seller will gain from the release of information not because of the linkage principle, but because the information can improve efficiency. As we show, some of the potential efficiency gain is appropriated by the seller.

Several papers have studied issues related to how buyers learn their valuations in auctions, and what consequences that bears on the seller's revenue. One strand of the literature, see Persico (2000), Compte and Jehiel (2001) and the references therein, focuses on the buyers' incentives to acquire information in different auction formats. Our approach is different in that we want to design a revenue-maximizing mechanism in which the seller has the opportunity to costlessly release (without observing) information to the buyers. In our model, it is the seller (not the buyers) who controls how much information the buyers acquire.

In Baron and Besanko (1984), Riordan and Sappington (1987), and Courty and Li (2000), a principal and an agent are contracting over two periods. Independently of the contract, the agent learns payoff-relevant private information in both periods. These papers analyze the optimal two-stage revelation mechanism where the contract is signed in the first period, when the agent only knows his first-period type. In contrast, in our paper, we solve for the optimal mechanism in a multi-agent auction environment where the seller can decide whether or not the buyers receive additional private signals.

A related literature is that on optimal entry fees in auctions (e.g., French and McCormick (1984), McAfee and McMillan (1987), Levin and Smith (1994)). In these papers, in contrast to ours, entry is endogenous, and competition drives down the bidders' payoffs to zero. As a result, the seller maximizes the total social surplus, and she 
levies (ex ante) entry fees in order to induce socially optimal entry. In the independent private values case, for example, socially optimal entry is achieved by setting entry fees to zero and committing to an efficient allocation. Our approach is different in that we focus on the complete mechanism design problem with a fixed number of bidders, and transfers are only allowed at the stage when bidders already have private information. Consequently, our results are also markedly different from those in this literature. Interpreting the transfers in our model as (interim) entry fees, we find that it is optimal for the seller to set them different from zero, and to induce ex-post inefficiency by sometimes not selling the good to the buyer with the highest true valuation.

Our motivation is closer to that of Bergemann and Pesendorfer (2002) and Ganuza (2003), where the seller decides how much private information the buyers may learn prior to participating in an auction. Ganuza (2003) focuses on the incentives of the auctioneer to release signals to the buyers that refine their private valuations before a second-price auction. ${ }^{4}$ He finds that, in order to maximize the revenue, the seller should not reveal all her information. In our model, we find just the opposite: the seller releases everything she can. The reason for the discrepancy is that Ganuza (2003) fixes the selling mechanism as a second-price auction, and the seller must reveal her information before the bidders can take any action. In contrast, we allow the seller to design the fully optimal mechanism. A feature of this auction is that the seller first elicits the private information of the bidders, and only then does she reveal her own. The main difference between our paper and that of Bergemann and Pesendorfer (2002) is that in their paper, the seller first designs a disclosure policy and reveals information, and only then proposes the selling mechanism. Moreover, the seller cannot commit between the two stages. In contrast, in our model the timing is simultaneous. ${ }^{5}$ Under their assumptions, Bergemann and Pesendorfer (2002) show that the information structure that allows the seller to subsequently design the auction with the largest expected revenue is necessarily imperfect. In this structure, buyers are only allowed to learn which element of a finite partition their valuation falls into.

Compared to these two papers, the difference of our approach is that we consider the design of the information structure and the transaction rules as one mechanism

\footnotetext{
${ }^{4}$ By allowing the buyers to have more private information, the seller faces a trade-off. More information increases efficiency and potentially the revenue, but it also increases the buyers' information rents in the second-price auction.

${ }^{5} \mathrm{~A}$ less important difference is that in Bergemann and Pesendorfer (2002) the buyers do not possess private information at the beginning of the game.
} 
design problem, as a whole. This difference may first seem subtle, nevertheless, it is important. In our model, the seller can integrate the rules of information acquisition into the mechanism used for the sale itself. For example, the seller can charge the buyers for getting more and more accurate signals (perhaps in several rounds); the buyers may even be asked to bid for obtaining more information. In contrast to the two papers cited above, we show that the seller maximizes expected revenue by designing a mechanism in which she allows the buyers to learn their valuations as precisely as they can. ${ }^{6}$ In other words, the tradeoff between allowing the buyers to obtain more private information and generating more revenue disappears in the optimal auction.

The idea that "selling" the access to information may be profitable is not new, and can be illustrated by an example. Suppose that there are two buyers who are unaware of their private valuations, which the seller can allow them to learn. Consider the following mechanism: The seller charges both buyers an entry fee. Then, she allows the buyers to observe their valuations, and makes them play a second-price auction with zero reservation price. The second-price auction will be efficient. If the entry fees equal the buyers' ex-ante expected profits then the seller appropriates the entire surplus. ${ }^{7}$

This simple solution - the seller committing to the efficient allocation, releasing the additional signals, and charging an entry fee equal to the expected efficiency gainsonly works when the buyers do not have private information to start with. Otherwise (for example, if the buyers privately observe signals, but their valuations also depend on other signals that they may see at the seller's discretion), the auctioneer, as we will show, does not want to commit to an efficient auction in the continuation, so the previously proposed mechanism does not work. One has to find a more sophisticated auction, and this is exactly what we will do in the remainder of the paper.

\footnotetext{
${ }^{6}$ Note that we, just like the existing literature, require the participation and incentive constraints to hold at all interim phases (not just ex ante), i.e., the buyers want to go ahead with the mechanism at every point in the game.

${ }^{7}$ The example is the basis of Gershkov (2002). Demsetz (1968) and Loeb and Magat (1979) proposed the same method - the ex ante sale of all future rents - for the efficient regulation of natural monopolies. In the context of a two-period principal-agent problem, Baron and Besanko (1984) showed that if the agent's type in the second period is independent of his type in the first period then a period one (constant and efficient) contract gives the whole period-two surplus to the principal.
} 


\section{The Model}

\subsection{The Environment}

There are $n$ potential buyers for a single indivisible good sold by a seller (she). All parties are risk neutral. The seller's valuation for the good is normalized to zero; her objective is to maximize the expected revenue. Each buyer's payoff is the negative of his payment to the seller, plus, in case he wins, the value of the object.

Buyer $i$ 's true valuation for the object is $V_{i}$. However, he only observes a noisy signal of it, $v_{i}$, which is his private information. The seller has the ability to disclose to buyer $i$, without observing, an additional noisy signal about $V_{i}$ that is denoted by $z_{i} .{ }^{8}$ Assume that $E\left[V_{i} \mid v_{i}, z_{i}\right]$ is strictly increasing in $z_{i}$. For example, $z_{i}$ may be identical to $V_{i}$, that is, the seller may be able to reveal to buyer $i$ his actual valuation for the good. We can also allow the seller to only partially reveal $z_{i}$ to buyer $i$, for example, by disclosing a signal positively correlated with $z_{i}$. However, in order to simplify the exposition (and without affecting the results), we will not formally model this possibility. ${ }^{9}$

Denote the distribution of buyer $i$ 's type, $v_{i}$, by $F_{i}$, and the corresponding density by $f_{i}$. We assume that $f_{i}$ is positive on the interval $\left[\underline{v}_{i}, \bar{v}_{i}\right]$, and satisfies the monotone hazard rate condition, that is, $f_{i} /\left(1-F_{i}\right)$ is weakly increasing.

We emphasize that buyer $i$ 's initial signal and the seller's signal, $v_{i}$ and $z_{i}$, need not be independent, nor even conditionally independent given $V_{i}$. On the other hand, the pairs $\left(v_{i}, z_{i}\right)$ are assumed to be independent across $i$. The independence of information across buyers is a standard assumption that rules out Crémer and McLean's (1988) full rent extracting mechanisms.

Since the buyers are risk neutral we can assume without loss of generality that $V_{i}=E\left[V_{i} \mid v_{i}, z_{i}\right]$, that is, after the seller reveals her signals, the buyers know their true valuations (e.g., $z_{i} \equiv V_{i}$ or $z_{i} \equiv V_{i}-v_{i}$ ). In other words, $V_{i}$ is the buyer's posterior valuation given his and the seller's signals. Let $H_{i v_{i}}$ denote the distribution of $V_{i}$ conditional on $v_{i}$, and $h_{i v_{i}}$ the corresponding density. ${ }^{10}$ We assume that if $v_{i}>\widehat{v}_{i}$

\footnotetext{
${ }^{8} \mathrm{An}$ alternative interpretation of the model is that the seller can in fact observe the signals that she releases, but she does not know how they affect the valuations of the buyers.

${ }^{9} \mathrm{We}$ will show in Theorem 1 that by fully revealing her signals, the seller can do as well as in a specific "benchmark" situation that is not attainable by partial revelation. Therefore, restricting attention to the case where the seller can decide to either reveal $z_{i}$ to buyer $i$ or not reveal it at all does not affect the results.

${ }^{10}$ The function $H_{i v_{i}}$ is assumed to be twice continously differentiable, strictly increasing on $\mathbb{R}$. This
} 
then $H_{i v_{i}}$ first-order stochastically dominates $H_{i \widehat{v}_{i}}$, that is, $\partial H_{i v_{i}} / \partial v_{i}<0$. We need the following two additional assumptions on the joint distribution of $V_{i}, z_{i}$, and $v_{i}$.

Assumption 1: $\left(\partial H_{i v_{i}}\left(V_{i}\right) / \partial v_{i}\right) / h_{i v_{i}}\left(V_{i}\right)$ is increasing in $V_{i}$.

Assumption 2: $\left(\partial H_{i v_{i}}\left(V_{i}\right) / \partial v_{i}\right) / h_{i v_{i}}\left(V_{i}\right)$ is increasing in $v_{i}$.

We shall argue that these assumptions can be interpreted as a kind of substitutability in buyer $i$ 's posterior valuation between $v_{i}$ and the part of $z_{i}$ that is new to buyer $i$. In order to explain these assumptions, and make it precise what we mean by new information, we perform an orthogonal decomposition of $z_{i}$ in the next subsection.

\subsection{Decomposition of the Seller's Signal}

Imagine that buyer $i$ 's type is $v_{i}$, and the seller can disclose to him, without observing, $s_{i}\left(z_{i}, v_{i}\right)$ instead of $z_{i}$, where $s_{i}$ is strictly monotonic in $z_{i}$. First, notice that since $s_{i}$ is monotonic, buyer $i$ can recover $z_{i}$ from $s_{i}\left(z_{i}, v_{i}\right)$ and execute the same Bayesian updating to compute his posterior valuation as before. Second, this change makes no difference for the seller either since she cannot observe $z_{i}$ or $s_{i}$ anyway. Hence, our model remains strategically the same. In particular, the seller's expected revenue is invariant to such a transformation of the signals.

In Lemma 1 we show that for each $v_{i}$ there exists a particular transformation of $z_{i}$ for which the random variable $s_{i}\left(z_{i}, v_{i}\right)$ is independent of $v_{i}$. Since $s_{i}$, which we call buyer $i$ 's shock, is orthogonal to the buyer's initial value estimate, it should be considered as the part of the seller-controlled information, $z_{i}$, that is new for the buyer.

Lemma 1 (i) There exist functions $u_{i}$ and $s_{i}$ such that $u_{i}\left(v_{i}, s_{i}\left(z_{i}, v_{i}\right)\right) \equiv V_{i}$, such that $u_{i}$ is strictly increasing, $s_{i}$ is strictly increasing in $z_{i}$, and $s_{i}\left(z_{i}, v_{i}\right)$ is independent of $v_{i}$.

(ii) All $s_{i}$ 's satisfying part (i) are positive monotonic transformations of each other.

Proof. (i) Define $s_{i}\left(z_{i}, v_{i}\right) \equiv H_{i v_{i}}\left(V_{i}\right)$, which is strictly increasing in $z_{i}$ by assumption. Note that for any $y \in[0,1], \operatorname{Pr}\left(H_{i v_{i}}(V) \leq y\right)=\operatorname{Pr}\left(V \leq H_{i v_{i}}^{-1}(y)\right)=H_{i v_{i}}\left(H_{i v_{i}}^{-1}(y)\right)=y$. That is, $s_{i}$ is uniform on $[0,1]$ irrespective of the value of $v_{i}$, and hence it is independent of $v_{i}$. Let $u_{i}\left(v_{i}, s_{i}\right)=H_{i v_{i}}^{-1}\left(s_{i}\right)$, which is by definition identical to $V_{i}$. The monotonicity of $u_{i}$ and part (ii) are proved in the Appendix.

assumption is purely for convenience. As a result, any realization of the ex-post valuation, $V_{i}$, is possible given any type $v_{i}$ of buyer $i$. 
In part (i) of the lemma, $s_{i}$ is constructed as the percentile of the distribution of $V_{i}$ conditional on $v_{i}$ that the realization of the true valuation belongs to. The function $u_{i}$ is the formula that buyer $i$ can use to compute his posterior valuation given his type $v_{i}$ and signal $s_{i}$. We will denote the cdf of $s_{i}$ by $G_{i} \cdot{ }^{11}$

The transformation in the proof of Lemma 1 requires no assumption regarding the joint distribution of $V_{i}, v_{i}$ and $z_{i}$. However, assumptions made on this distribution translate into properties of the function $u_{i}$. For example, since $h_{v}>0$ on $\mathbb{R}$ (see Footnote 10), for all $v_{i}, s_{i}$ and $v_{i}^{\prime}$, there exists $s_{i}^{\prime}$ such that $u_{i}\left(v_{i}, s_{i}\right)=u_{i}\left(v_{i}^{\prime}, s_{i}^{\prime}\right)$.

Now we show what Assumptions 1 and 2 imply regarding the shape of $u_{i}$. Denote the partial derivatives of $u_{i}$ by $u_{i 1}=\partial u_{i} / \partial v_{i}, u_{i 2}=\partial u_{i} / \partial s_{i}, u_{i 11}=\partial^{2} u_{i} / \partial v_{i}^{2}$, and $u_{i 12}=\partial^{2} u_{i} / \partial v_{i} \partial s_{i}$. The statement of the next lemma does not depend on the choice of $s_{i}$ and $u_{i}$, as long as they satisfy part (i) of Lemma 1 .

Lemma 2 Assumption 1 is equivalent to $u_{i 12} \leq 0$, and Assumption 2 is equivalent to $u_{i 11} / u_{i 1} \leq u_{i 12} / u_{i 2}$.

Proof. See the Appendix.

We are ready to interpret Assumptions 1 and 2. Assumption 1 says that the marginal impact of the $s_{i}$ shock on buyer $i$ 's valuation is non-increasing in his type, $v_{i}$. Assumption 2 means that an increase in $i$ 's type, holding the ex-post valuation constant, weakly decreases the marginal value of $v_{i}{ }^{12}$ The assumptions imply a certain monotonicity condition that is sufficient for our results. We will comment on the necessity of these assumptions in Section 4.3, after we present the results.

It is interesting to note that our assumptions are easier to interpret in terms of $u_{i}$, as decreasing-returns and substitutability conditions on $v_{i}$ and $s_{i}$, than in terms of the correlations between $V_{i}, v_{i}$ and $z_{i}$. Intuitively, in order to determine the information rents that the seller has to leave with the buyers in the optimal mechanism, what matters is not how the "raw" signals are correlated (with each other and the buyers' actual values), instead, what matters is whether or not the buyers' original information and the "new" part of the seller's signals are substitutes in the buyers' ex-post valuations.

\footnotetext{
${ }^{11}$ Notice that the $s_{i}$ constructed in part (i) of Lemma 1 is uniform on $[0,1]$, therefore $G_{i}$ could be assumed to be uniform on $[0,1]$.

${ }^{12}$ To see this interpretation of Assumption 2 note that the total differential of $u_{i 1}$ (the change in the marginal value of $i$ 's type) is $u_{i 11} d v_{i}+u_{i 12} d s_{i}$. Keeping $u_{i}$ constant (moving along an "isovalue" curve) means $d s_{i}=-u_{i 1} / u_{i 2} d v_{i}$. Substituting this into the total differential of $u_{i 1}$ yields $\left(u_{i 11}-u_{i 12} u_{i 1} / u_{i 2}\right) d v_{i}$. This expression is non-positive for $d v_{i}>0$ if and only if $u_{i 11} / u_{i 1} \leq u_{i 12} / u_{i 2}$.
} 


\subsection{Applications, Examples}

In order to give a flavor of the type of situations that our model covers we now provide a few applications and examples where our assumptions hold.

Example 1. Assume that buyer $i$ 's true valuation for the good differs from his type by an additive and independent noise: $V_{i}=v_{i}+z_{i}$, where $z_{i}$ is independent of $v_{i}$. Suppose that the seller can resolve the uncertainty in buyer $i$ 's valuation by disclosing, without observing, $z_{i}$. Since $z_{i}$ is independent of $v_{i}$, the buyer's original private information pertains only to the expected value of the good. Among other things, $v_{i}$ conveys no information regarding the precision of the buyer's estimate. Assume that all signals are independent across $i$ 's and that the distribution of $v_{i}$ satisfies the monotone hazard rate condition. The transformed model obtains by setting $s_{i} \equiv z_{i}$ and $u_{i}\left(v_{i}, s_{i}\right)=v_{i}+s_{i}$. By the linearity of $u_{i}$, Assumptions 1 and 2 hold.

For a specific example, suppose that the object for sale is a car, and assume that the buyer knows its make, model, age and mileage, but not its color, which the seller can reveal. It seems reasonable to assume that a buyer's initial willingness to pay for the car $\left(v_{i}\right)$ and his color preference $\left(z_{i}\right)$ are statistically independent.

Example 2a. Consider the following familiar "normal sampling" problem. Suppose that buyer $i$ 's true valuation for the good, $V_{i}$, is drawn from a normal distribution with mean $\mu_{i}$ and precision (inverse variance) $\tau_{i 0}$. His signal, $v_{i}$, is normally distributed with mean $V_{i}$ and precision $\tau_{i v}{ }^{13}$ Suppose that the seller can allow buyer $i$ to observe his true valuation, that is, $z_{i} \equiv V_{i}$. Clearly, $V_{i}, z_{i}$ and $v_{i}$ are strictly affiliated. The distribution of $v_{i}$, which is normal, satisfies the hazard rate condition.

The cdf of $V_{i}$ conditional on $v_{i}, H_{i v_{i}}$, is normal with mean $\left(\tau_{i 0} \mu_{i}+\tau_{i v} v_{i}\right) /\left(\tau_{i 0}+\tau_{i v}\right)$ and precision $\tau_{i 0}+\tau_{i v}$. The realization of $v_{i}$ simply "shifts" the conditional distribution of $V_{i}$ to the right without altering its shape: For a unit increase in $v_{i}$, the mean (and only the mean) of $i$ 's true valuation increases by $\tau_{i v} /\left(\tau_{i 0}+\tau_{i v}\right)$.

In order to show that this example fits our model, we just need to verify Assumptions 1 and 2. The easiest way to do so is by transforming the seller's signal and showing that the conditions in Lemma 2 hold. Define $s_{i} \equiv H_{i v_{i}}\left(z_{i}\right)$, and let $u_{i}\left(v_{i}, s_{i}\right)=H_{i v_{i}}^{-1}\left(s_{i}\right) \equiv V_{i}$. It is immediate that $u_{i}$ is strictly increasing in $s_{i}$. Since $v_{i}$ shifts $H_{i v_{i}}$ (as a function of $\left.z_{i}\right)$ to the right by $\tau_{i v} /\left(\tau_{i 0}+\tau_{i v}\right)$ for a unit increase in $v_{i}$, the derivative of $H_{i v_{i}}^{-1}$ with

\footnotetext{
${ }^{13}$ The support of $v_{i}$ is not a compact interval, but this does not cause a problem in the analysis.
} 
respect to $v_{i}$ is constant, $\tau_{i v} /\left(\tau_{i 0}+\tau_{i v}\right)$. Therefore $u_{i}$ is linear and strictly increasing in $v_{i}$, and Assumptions 1 and 2 are satisfied.

Example 2a generalizes to the case where $z_{i}$, just like $v_{i}$, is a noisy, normal signal of $V_{i}$ (details available upon request). In Examples 1 and $2 \mathrm{a}$, in the transformed model $u_{i}$ is linear in $v_{i}$. We will show in Section 5 that the implementation of the optimal mechanism is particularly simple in this case. However, our model applies more generally.

Example 2b. Suppose that the environment is the same as in Example 2a, with the only difference that the random variable $V_{i}$ is not the monetary value of the good for buyer $i$; instead, it is the quantity of an input that the buyer obtains by buying the good. (Think of the auctioned good as a mineral field and $V_{i}$ as the quantity of the raw material in the field.) Suppose that the buyer's monetary gain from owning the good is an increasing, continuously differentiable and concave function of $V_{i}$, say, $r_{i}\left(V_{i}\right)$. The buyer is risk neutral towards monetary gains and losses.

Set, for example, $r_{i}\left(V_{i}\right)=V_{i}$ for $V_{i} \leq 0$ and $r_{i}\left(V_{i}\right)=2\left(\sqrt{V_{i}+1}-1\right)$ for $V_{i} \geq 0$. Then, in the transformed model, the signal $s_{i}$ is the same as in the transformed version of Example 2a, and the buyer's monetary valuation for the good is $\tilde{u}_{i}\left(v_{i}, s_{i}\right) \equiv r_{i}\left(u_{i}\left(v_{i}, s_{i}\right)\right)$. This function is clearly nonlinear, yet it also satisfies the conditions in Lemma 2 because $u_{i}$ is linear in $v_{i}$ and $r_{i}$ is concave.

In the following example $u_{i}$ is genuinely nonlinear but our model applies.

Example 3. Suppose that the joint distribution of $v_{i}$ and $V_{i}$ has a pdf $f\left(v_{i}, V_{i}\right)=2 V_{i}$ for $v_{i} \in[0,1]$ and $V_{i} \in\left[\sqrt{v_{i}}, \sqrt{1+v_{i}}\right]$. The buyer's signal, $v_{i}$, is uniform on $[0,1]$ :

$$
f_{v}\left(v_{i}\right)=\int_{\sqrt{v_{i}}}^{\sqrt{1+v_{i}}} 2 V_{i} d V_{i}=\left[V_{i}^{2}\right]_{\sqrt{v_{i}}}^{\sqrt{1+v_{i}}}=\left(\sqrt{1+v_{i}}\right)^{2}-\left(\sqrt{v_{i}}\right)^{2}=1 .
$$

The pdf of $V_{i}$ conditional on $v_{i}$ is $f_{V \mid v}\left(V_{i} \mid v_{i}\right)=f\left(v_{i}, V_{i}\right) / f_{v}\left(v_{i}\right)=2 V_{i}$, therefore the conditional cdf is

$$
F_{V \mid v}\left(V_{i} \mid v_{i}\right)=\int_{\sqrt{v_{i}}}^{V_{i}} f_{V \mid v}\left(t \mid v_{i}\right) d t=\int_{\sqrt{v_{i}}}^{V_{i}} 2 t d t=\left[t^{2}\right]_{\sqrt{v_{i}}}^{V_{i}}=V_{i}^{2}-v_{i} .
$$

Define $s_{i}=F_{V \mid v}\left(V_{i} \mid v_{i}\right)$, and let $u_{i}\left(v_{i}, s_{i}\right)=\sqrt{v_{i}+s_{i}} \equiv V_{i}$. By direct calculation $u_{i 1}=$ $u_{i 2}=\left(v_{i}+s_{i}\right)^{-1 / 2} / 2$ and $u_{i 11}=u_{i 12}=-\left(v_{i}+s_{i}\right)^{-3 / 2} / 4$. Therefore $u_{i 12}<0$, satisfying Assumption 1, and $u_{i 11} / u_{i 1}=u_{i 12} / u_{i 2}$, satisfying Assumption 2. The distribution of $v_{i}$ is uniform, which satisfies the hazard rate condition. 


\section{Preview of the Results for a Single Buyer}

In this section we preview our results for the case of a single buyer in the context of Example 2a. This special case allows us to outline the main results of the paper and some heuristic proofs. It also showcases the practical aspects of the optimal mechanism and its relation to mechanisms observed in reality, in particular, option contracts. ${ }^{14}$

In Example 2a the buyer's valuation for the object on sale, $V$, is normally distributed with mean $\mu$ and variance $1 / \tau_{0}$. The buyer only observes the signal $v$, whose distribution conditional on $V$ is normal with mean $V$ and variance $1 / \tau_{v}$. The unconditional distribution of $v$ is normal with mean $\mu$ and variance $1 / \tau_{0}+1 / \tau_{v}$, hence the cdf of $v, F$, satisfies the hazard rate condition. The conditional distribution of $V$ given $v$, $H_{v}$, is also normal with mean $\left(\tau_{0} \mu+\tau_{v} v\right) /\left(\tau_{0}+\tau_{v}\right)$ and variance $1 /\left(\tau_{0}+\tau_{v}\right)$. The seller can disclose to the buyer, without observing, the realization of $V$. We want to find the optimal mechanism that incorporates the rules of information disclosure and sale.

The main difficulty of this mechanism design problem is that in this setup, it seems impossible to characterize all feasible mechanisms. For example, it is clearly not without loss of generality to assume that the seller discloses $V$ and asks the buyer to report it back. Our approach will be to analyze the optimal solution in a relaxed problem and prove that it is implementable in the original environment.

Suppose that the agents have access to a computer (black box) that works as follows: If the buyer privately inputs $v$ and the seller reveals to the machine (without observing) $V$ then it outputs $H_{v}(V)$. That is, the machine computes the percentile of the distribution of $V$ given $v$ that the realization of the true valuation belongs to. Call the output of the machine (which is a random variable) the signal $s$. In the proof of part (i) of Lemma 1 we established that $s \equiv H_{v}(V)$ is distributed uniformly on $[0,1]$ for all realizations of $v$, hence it is independent of $v$.

It does not matter for the buyer whether he observes $V$ or $s$; knowing $v$, he can compute $V=H_{v}^{-1}(s)$. However, the seller would be better off if she could commit to a mechanism contingent on $s$ before observing $s$ as she can always discard the information. The benchmark case is the hypothetical situation where the seller commits to a mechanism that may depend on $s$ and then observes $s$ (but not $V$ or $v$ ). The seller's expected revenue in the benchmark is an upper bound on that in the original problem.

\footnotetext{
${ }^{14}$ We thank the Editor, Juuso Välimäki, for suggesting that we include this section and point out the realistic features of the optimal mechanism.
} 
The benchmark case can be analyzed using standard tools of mechanism design. In particular, any feasible mechanism can be represented by a truthful direct mechanism, where the buyer is asked to reveal $v$, the seller observes $s$, trade takes place with probability $X(v, s)$, and the buyer pays an expected transfer of $T(v, s)$. If the buyer reports type $\hat{v}$ while his actual type is $v$, his payoff in the mechanism is

$$
\pi^{*}(v, \hat{v})=\int_{0}^{1}\left[H_{v}^{-1}(s) X(\hat{v}, s)-T(\hat{v}, s)\right] d s .
$$

The mechanism is incentive compatible if $\pi^{*}(v, \hat{v})$ is maximized in $\hat{v}$ at $\hat{v}=v$. The first-order condition of maximization is $\partial \pi^{*}(v, \hat{v}) / \partial \hat{v}=0$ at $\hat{v}=v$, the second-order condition boils down to $\int_{0}^{1} X(v, s) d s$ being increasing in $v$ (strictly for sufficiency).

By the Envelope Theorem, $d \pi^{*}(v, v) / d v=\partial \pi^{*}(v, \hat{v}) / \partial v$ at $\hat{v}=v$. In Example 2a we saw that $\partial H_{v}^{-1}(s) / \partial v=\tau_{v} /\left(\tau_{0}+\tau_{v}\right)$, a constant that we will denote by $\lambda$. Therefore, $d \pi^{*}(v, v) / d v=\int_{0}^{1} \lambda X(v, s) d s$. Integrating it in $v$ and using (1) yields,

$$
\pi^{*}(v, v)=\underline{\pi}+\int_{\underline{v}}^{v} \int_{0}^{1} \lambda X(y, s) d s d y
$$

where $\underline{v}$ is the infimum of types that receive the good with positive probability, and $\underline{\pi}$ is this type's payoff. The ex-ante expectation of the buyer's surplus is,

$$
\int_{-\infty}^{\infty} \pi^{*}(v, v) d F(v)=\underline{\pi}+\int_{-\infty}^{\infty}\left[\int_{0}^{1} \lambda X(v, s) d s \frac{1-F(v)}{f(v)}\right] d F(v),
$$

where we used integration by parts (or Fubini's theorem) in the second term.

The seller's expected payoff is the difference between social surplus and the buyer's expected surplus, which by the previous expression can be written as

$$
W=\int_{-\infty}^{\infty} \int_{0}^{1}\left(H_{v}^{-1}(s)-\lambda \frac{1-F(v)}{f(v)}\right) X(v, s) d s d F(v)-\underline{\pi} .
$$

The seller maximizes $W$ by choosing $X$ and $\underline{\pi}$. The constraints to her problem are: (i) $X \in[0,1]$, (ii) $\int_{0}^{1} X(v, s) d s$ weakly increasing in $v$, and (iii) $\underline{\pi} \geq 0$. The optimum is attained by setting $\underline{\pi}=0$, and maximizing the integrand in (3) pointwise by letting

$$
X(v, s)= \begin{cases}1 & \text { if } H_{v}^{-1}(s)-\lambda(1-F(v)) / f(v) \geq 0, \\ 0 & \text { otherwise. }\end{cases}
$$


The resulting $X$ is between 0 and 1, it is strictly increasing in $v$ as $H_{v}^{-1}$ is increasing and $(1-F) / f$ is decreasing in $v$. The constraints are satisfied, and we have an optimum.

Equation (4) defines the allocation rule of the optimal mechanism in the benchmark case. According to it, the buyer receives the object exactly when his true valuation, $V \equiv H_{v}^{-1}(s)$, exceeds the threshold $\lambda(1-F(v)) / f(v)$. By plugging (4) into (3), the seller's expected revenue in the optimal mechanism can be written as

$$
W=\int_{-\infty}^{\infty} \int_{0}^{1} \max \left\{H_{v}^{-1}(s)-\lambda \frac{1-F(v)}{f(v)}, 0\right\} d s d F(v)
$$

The interpretation of the optimal mechanism in the benchmark is familiar from Bulow and Roberts (1989): The seller's marginal revenue from the buyer with type $v$ and valuation $V$ is $V-\lambda(1-F(v)) / f(v)$. The object is sold whenever this expression is non-negative, and the total revenue is the area under the marginal revenue curve.

Our main result is that the seller can implement the same outcome in the original model even without observing $s$. The intuition is that the seller's marginal revenue from buyer type $v$ is $V-\lambda(1-F(v)) / f(v)$ in the original model as well, at least, as long as the seller allows the buyer to learn $V$. However, this is not the entire argument: it turns out that the conditions under which the same allocation rule can be implemented without the seller observing $s$ are stronger than they are in the benchmark model. In what follows, we provide a more formal analysis.

Consider mechanisms where the buyer first reports his type, $v$, and pays a fee $c(v)$. Then, the seller allows him to observe $V$. The buyer makes the decision whether or not to buy; if he buys, he pays an additional premium $p(v)$. (The functions $c$ and $p$ are announced by the seller before $v$ is reported.) We argue that such a mechanism can implement the outcome of the benchmark, (4)-(5).

The buyer with type $v$ who initially announces $\hat{v}$ buys the good in the end if and only if $V \geq p(\hat{v})$, because $c(\hat{v})$, which is paid no matter whether or not he buys, is sunk. If the seller sets $p(v)=\lambda(1-F(v)) / f(v)$ then the buyer who reports $v$ truthfully gets the good if and only if $V \geq \lambda(1-F(v)) / f(v)$, which is exactly the allocation rule in the benchmark according to equation (4). Now we define a fee schedule $c(v)$ that, together with $p(v)$ defined above, elicits a truthful type announcement. By reporting 
$\hat{v}$, the buyer with signal $v$ gets a payoff of

$$
\pi(v, \hat{v})=\int_{0}^{1} \max \left\{H_{v}^{-1}(s)-p(\hat{v}), 0\right\} d s-c(\hat{v})
$$

The first-order condition of incentive compatibility is $\partial \pi(v, \hat{v}) / \partial \hat{v}=0$ at $\hat{v}=v$, that is,

$$
c^{\prime}(v)=-p^{\prime}(v)\left[1-H_{v}(p(v))\right]
$$

The necessary second-order condition of the maximization is $p^{\prime} \leq 0$, which holds strictly (implying sufficiency) for $p=\lambda(1-F) / f$ by the hazard rate condition. ${ }^{15}$ Equation (7) defines $c$ up to a constant, which is set so that for an arbitrary $v, \pi(v, v)=\pi^{*}(v, v)$.

This mechanism implements the allocation rule of the benchmark. We only need to prove that the seller gets the same expected payoff as well. By the definition of $c$, $\pi(v, v)=\pi^{*}(v, v)$ for some $v$. By differentiating (2), and using (4),

$$
\frac{d}{d v} \pi^{*}(v, v)=\int_{0}^{1} X(v, s) d s=\lambda\left[1-H_{v}(\lambda(1-F(v)) / f(v))\right] .
$$

Using (7), the total derivative of $\pi(v, v)$ becomes

$$
\frac{d}{d v} \pi(v, v)=\int_{H_{v}(p(v))}^{1}\left(\lambda-p^{\prime}(v)\right) d s-c^{\prime}(v)=\lambda\left[1-H_{v}(p(v))\right] .
$$

Since $p=\lambda(1-F) / f$, we have $d \pi(v, v) / d v=d \pi^{*}(v, v) / d v$ for all $v$. We conclude that the buyer's payoff is the same under the proposed mechanism and the benchmark: $\pi(v, v)=\pi^{*}(v, v)$ for all $v$. The seller's revenue is the difference between the social surplus and the buyer's payoff. In the proposed mechanism, the buyer's and the social surpluses are the same as their respective counterparts in the benchmark. Therefore, the seller attains the same expected revenue in both cases.

The mechanism consisting of the pair of functions $(c, p)$ is remarkably simple, and can be thought of as a menu of European call options offered by the seller. These types of options are widely used in financial forward markets, and in labor and other productions contracts as well. (Prominent examples include professional athletes' labor

\footnotetext{
${ }^{15}$ Recall that in the benchmark case, the corresponding second-order condition required that $\int_{0}^{1} X^{*}(v, s) d s$ be increasing in $v$, which held because $H_{v}^{-1}(s)-\lambda(1-F(v)) / f(v)$ is increasing in $v$. In order to implement the same allocation rule in the original model, the local second-order condition is stronger: it requires that $p=\lambda(1-F) / f$ be decreasing in $v$.
} 
contracts and movie producer's option contracts regarding sequels.) In our model, it is the monopolist seller, not "time", that reveals new information to the buyer of the asset. However, this distinction makes no difference because the seller reveals all her information anyway. In the optimal mechanism, the buyer picks a fee, $c(v)$, depending on his prior, from a list provided by the seller. After having learned his posterior, the buyer has to pay a corresponding additional strike-price, $p(v)$, in case he decides to buy the good. Since $p=(1-F) / f$ is decreasing in $v$, higher buyer-types pick options with lower strike prices but larger upfront fees. (In financial markets, a European call option on the same future asset also costs more if the strike price is lower.) In our model, the reason why different buyers may choose options with different strike prices is that they have heterogeneous initial estimates regarding the asset's future value.

\section{The Optimal Mechanism in the General Case}

We now turn to the characterization of the expected revenue maximizing mechanism in the general model. Recall that we showed in Section 2.2 that the seller's signal can be transformed so that the resulting random variable, $s_{i}$ (buyer $i$ 's shock), is independent of $v_{i}$, and for an increasing function $u_{i}, V_{i} \equiv u_{i}\left(v_{i}, s_{i}\right)$. In Section 4.1 we characterize the optimal mechanism under the assumption that the seller, after having committed to a mechanism, is able to observe the realizations of the shocks. This provides an upper bound on her revenue in the original model as the seller can commit to ignore the shocks Then, in Section 4.2, we show that the same outcome can be implemented even if the seller cannot observe the $s_{i}$ 's, but controls their release. While the buyers still enjoy information rents from their types, all their rents from observing the seller-controlled signals are appropriated by the seller. This is the main result of the paper.

\subsection{Benchmark: The Seller Can Observe the Shocks}

Suppose first, for benchmarking purposes only, that the seller can observe the $s_{i}$ 's after having committed to a selling mechanism. ${ }^{16}$ The Revelation Principle applies, hence we can restrict attention to mechanisms where the buyers report their types, and the seller

\footnotetext{
${ }^{16}$ Since the seller observes the shocks only after having committed to a mechanism, "informed principal" type problems do not arise in the benchmark. It does not matter whether the buyers can observe the shocks as long as the mechanism is verifiable (i.e., the seller cannot lie about $s_{i}$ ).
} 
determines the allocation and the transfers as a function of the reported types and the realization of the shocks. ${ }^{17}$ We will analyze truthful equilibria of direct mechanisms that consist of an allocation rule, $x_{i}\left(v_{i}, v_{-i}, s_{i}, s_{-i}\right)$ for all $i$, and an (expected) transfer scheme, $t_{i}\left(v_{i}, v_{-i}, s_{i}, s_{-i}\right)$ for all $i$. Here, $x_{i}\left(v_{i}, v_{-i}, s_{i}, s_{-i}\right)$ is the probability that buyer $i$ receives the good, and $t_{i}\left(v_{i}, v_{-i}, s_{i}, s_{-i}\right)$ is the transfer that he expects to pay, given the reported types and the realization of the shocks.

We shall prove that the revenue maximizing allocation is in a way a generalization of the one in Myerson (1981). The object is rewarded to the bidder with largest nonnegative "shock-adjusted virtual valuation," $W_{i}\left(v_{i}, s_{i}\right)$, where

$$
W_{i}\left(v_{i}, s_{i}\right)=u_{i}\left(v_{i}, s_{i}\right)-\frac{1-F_{i}\left(v_{i}\right)}{f_{i}\left(v_{i}\right)} u_{i 1}\left(v_{i}, s_{i}\right) .
$$

Ignoring ties, the optimal allocation rule $x_{i}^{*}$ is defined as follows:

$$
x_{i}^{*}\left(v_{i}, v_{-i}, s_{i}, s_{-i}\right)=1 \text { if } i=\arg \max _{j}\left\{W_{j}\left(v_{j}, s_{j}\right), 0\right\} .
$$

Using the tools of Bayesian mechanism design, we obtain the following solution to the benchmark problem.

Proposition 1 In the revenue-maximizing mechanism of the benchmark case (when the seller can observe the $s_{i}$ 's after having committed to a selling mechanism), the allocation rule is defined by (9). The profit of buyer $i$ with type $v_{i}$ is

$$
\Pi_{i}^{*}\left(v_{i}\right)=\int_{\underline{v}_{i}}^{v_{i}} \int u_{i 1}\left(y, s_{i}\right) X_{i}^{*}\left(y, s_{i}\right) d G_{i}\left(s_{i}\right) d y,
$$

where $X_{i}^{*}\left(v_{i}, s_{i}\right)=\iint x_{i}^{*}\left(v_{i}, v_{-i}, s_{i}, s_{-i}\right) d F_{-i}\left(v_{-i}\right) d G_{-i}\left(s_{-i}\right)$. The seller's revenue is

$$
R^{*}=\iint \max _{i}\left\{u_{i}\left(v_{i}, s_{i}\right)-\frac{1-F_{i}\left(v_{i}\right)}{f_{i}\left(v_{i}\right)} u_{i 1}\left(v_{i}, s_{i}\right), 0\right\} d F(v) d G(s) .
$$

Proof. See the Appendix.

It is useful, for use in subsequent steps of the analysis, to further describe some properties of the allocation rule.

\footnotetext{
${ }^{17}$ Using standard shorthand notation, $v_{-i}$ and $s_{-i}$ denote the vector of types and shocks of buyers other than $i$, while $v$ and $s$ denote the entire profiles.
} 
Corollary 1 (i) $X_{i}^{*}$ is continuous in both of its arguments.

(ii) $X_{i}^{*}$ is weakly increasing in both of its arguments.

(iii) If $v_{i}>\hat{v}_{i}, s_{i}<\hat{s}_{i}$ and $u_{i}\left(v_{i}, s_{i}\right)=u_{i}\left(\hat{v}_{i}, \hat{s}_{i}\right)$ then $X_{i}^{*}\left(v_{i}, s_{i}\right) \geq X_{i}^{*}\left(\hat{v}_{i}, \hat{s}_{i}\right)$.

Proof. See the Appendix.

\subsection{The Solution to the Seller's Problem}

We now show that the allocation rule and seller's revenue characterized in Proposition 1 can be implemented even if the seller cannot observe the shocks, as long as she can allow the buyers to observe them. In order to implement the allocation rule (9), she has to reveal her signals to the buyers (otherwise nobody can compute $W_{i}$ ). Therefore, in order to find a mechanism that implements the benchmark allocation rule, we can restrict attention to two-stage, incentive-compatible direct mechanisms. In the first stage buyers report their $v_{i}$ 's; in the second stage each buyer observes his own $s_{i}$ and reports it back. For all reporting profiles $(v, s)$ and for all $i$, the seller allocates the good to buyer $i$ with probability $x_{i}\left(v_{i}, v_{-i}, s_{i}, s_{-i}\right)$, and buyer $i$ pays $t_{i}\left(v_{i}, v_{-i}, s_{i}, s_{-i}\right)$; these functions are set so that truth-telling is incentive compatible in both reporting stages.

Given a two-stage mechanism $\left\{x_{i}, t_{i}\right\}_{i=1}^{n}$, define

$$
\begin{aligned}
X_{i}\left(v_{i}, s_{i}\right) & =\iint x_{i}\left(v_{i}, v_{-i}, s_{i}, s_{-i}\right) d F_{-i}\left(v_{-i}\right) d G_{-i}\left(s_{-i}\right), \\
T_{i}\left(v_{i}, s_{i}\right) & \equiv \iint t_{i}\left(v_{i}, v_{-i}, s_{i}, s_{-i}\right) d F_{-i}\left(v_{-i}\right) d G_{-i}\left(s_{-i}\right),
\end{aligned}
$$

as buyer $i$ 's expected probability of winning and expected transfers, respectively.

We will now analyze the consequences of incentive compatibility going backwards, starting in the second stage of the mechanism. In Lemma 3 we show how the allocation rule pins down the buyers' second round profit functions given truthful revelation of types in the first round. Then, in Lemma 4 we describe what happens off the equilibrium path - after buyer $i$ reports his type untruthfully and observes his shock. It turns out that the deviator will report an untruthful value for his shock in such a way that the two lies "cancel" each other, and his true valuation, $u_{i}\left(v_{i}, s_{i}\right)$, is correctly inferred from his reports. Using these results, in Lemmas 5 and 6 (the final steps before proving Theorem 1), we derive the indirect profit function of buyer $i$.

In the second reporting stage, after truthful first round, buyer $i$ with type $v_{i}$ who 
observes $s_{i}$ and reports $\hat{s}_{i}$ gets

$$
\tilde{\pi}_{i}\left(s_{i}, \hat{s}_{i} ; v_{i}\right)=u_{i}\left(v_{i}, s_{i}\right) X_{i}\left(v_{i}, \hat{s}_{i}\right)-T_{i}\left(v_{i}, \hat{s}_{i}\right)
$$

Incentive compatibility in the second reporting stage is equivalent to

$$
\tilde{\pi}_{i}\left(s_{i}, \hat{s}_{i} ; v_{i}\right) \leq \tilde{\pi}_{i}\left(s_{i}, s_{i} ; v_{i}\right) \text { for all } i, v_{i}, s_{i} \text {, and } \hat{s}_{i} .
$$

The following lemma provides the conditions for a mechanism to be incentive compatible in the second reporting stage after a truthful first round.

Lemma 3 If a two-stage mechanism is incentive compatible and $X_{i}\left(v_{i}, s_{i}\right)$ induced by the allocation rule is continuous in $s_{i}$ then for all $s_{i}>\hat{s}_{i}$,

$$
\tilde{\pi}_{i}\left(s_{i}, s_{i} ; v_{i}\right)-\tilde{\pi}_{i}\left(\hat{s}_{i}, \hat{s}_{i} ; v_{i}\right)=\int_{\hat{s}_{i}}^{s_{i}} u_{i 2}\left(v_{i}, z\right) X_{i}\left(v_{i}, z\right) d z .
$$

Moreover, if (14) holds and $X_{i}$ is weakly increasing in $s_{i}$ then the two-stage mechanism is incentive compatible in the second round after truthful revelation in the first round.

Proof. See the Appendix.

In order to complete the analysis of the second round of the mechanism, we also need to know what buyer $i$ will do if he misreports his type in the first round. The following lemma claims that he will "correct his lie." 18

Lemma 4 If a two-stage mechanism is incentive compatible in the second round then buyer $i$ with type $v_{i}$ who reported $\hat{v}_{i}$ in the first round and observes $s_{i}$ reports $\hat{s}_{i}=$ $\sigma_{i}\left(v_{i}, \hat{v}_{i}, s_{i}\right)$ such that

$$
u_{i}\left(v_{i}, s_{i}\right) \equiv u_{i}\left(\hat{v}_{i}, \sigma_{i}\left(v_{i}, \hat{v}_{i}, s_{i}\right)\right) .
$$

Proof. Had buyer $i$ indeed have type $\hat{v}_{i}$ (as reported) and observed $\hat{s}_{i}$, incentive compatibility in the second round would require

$$
u_{i}\left(\hat{v}_{i}, \hat{s}_{i}\right) X_{i}\left(\hat{v}_{i}, \hat{s}_{i}\right)-T_{i}\left(\hat{v}_{i}, \hat{s}_{i}\right) \geq u_{i}\left(\hat{v}_{i}, \hat{s}_{i}\right) X_{i}\left(\hat{v}_{i}, s_{i}^{\prime}\right)-T_{i}\left(\hat{v}_{i}, s_{i}^{\prime}\right)
$$

\footnotetext{
${ }^{18}$ This correction is possible because for all $v_{i}, \hat{v}_{i}$, and $s_{i}$ there exists $\hat{s}_{i}$ such that $u_{i}\left(v_{i}, s_{i}\right)=u_{i}\left(\hat{v}_{i}, \hat{s}_{i}\right)$. If this assumption did not hold then the seller could detect deviations more easily.
} 
for all $s_{i}^{\prime}$. By $(15)$, that is $u_{i}\left(v_{i}, s_{i}\right)=u_{i}\left(\hat{v}_{i}, \hat{s}_{i}\right)$, this is equivalent to

$$
u_{i}\left(v_{i}, s_{i}\right) X_{i}\left(\hat{v}_{i}, \hat{s}_{i}\right)-T_{i}\left(\hat{v}_{i}, \hat{s}_{i}\right) \geq u_{i}\left(v_{i}, s_{i}\right) X_{i}\left(\hat{v}_{i}, s_{i}^{\prime}\right)-T_{i}\left(\hat{v}_{i}, s_{i}^{\prime}\right)
$$

which means that type $v_{i}$ that reported $\hat{v}_{i}$ in the first round and then observed $s_{i}$ is indeed best off by reporting $\hat{s}_{i}$.

Now we move back to the first round and examine the consequences of incentive compatibility there. Our goal is to derive the buyers' equilibrium profit functions, and we proceed as follows. Using the result of Lemma 4 regarding continuation play after a first-round deviation (buyer $i$ misreporting his type) we first derive the deviating buyer's profit function (see Lemma 5). In Lemma 6 we use these formulas for the deviator's payoff to derive the buyers' equilibrium (or indirect) profit functions.

Lemma 5 If a two-stage mechanism is incentive compatible in the second stage then $\pi_{i}\left(v_{i}, \hat{v}_{i}\right):=\int \tilde{\pi}_{i}\left(s_{i}, \sigma_{i}\left(v_{i}, \hat{v}_{i}, s_{i}\right) ; v_{i}\right) d G_{i}\left(s_{i}\right)$, buyer $i$ 's payoff with type $v_{i}$ reporting $\hat{v}_{i}$ in the first stage, can be written equivalently as

$$
\pi_{i}\left(v_{i}, \hat{v}_{i}\right)=\pi_{i}\left(\hat{v}_{i}, \hat{v}_{i}\right)+\iint_{\hat{v}_{i}}^{v_{i}} u_{i 1}\left(y, s_{i}\right) X_{i}\left(\hat{v}_{i}, \sigma_{i}\left(y, \hat{v}_{i}, s_{i}\right)\right) d y d G_{i}\left(s_{i}\right)
$$

where $\int_{a}^{b}$ denotes $-\int_{b}^{a}$ for $a<b$.

Proof. See the Appendix.

Incentive compatibility in the first round is equivalent to, for all $v_{i}>\hat{v}_{i}$,

$$
\pi_{i}\left(v_{i}, \hat{v}_{i}\right) \leq \pi_{i}\left(v_{i}, v_{i}\right) \text { and } \pi_{i}\left(\hat{v}_{i}, v_{i}\right) \leq \pi_{i}\left(\hat{v}_{i}, \hat{v}_{i}\right)
$$

Equation (16) is used in the following lemma to characterize the buyers' indirect profit functions in an incentive compatible two-stage mechanism.

Lemma 6 If a two-stage mechanism is incentive compatible and $X_{i}\left(v_{i}, s_{i}\right)$ induced by the allocation rule is continuous then buyer $i$ 's profit, $\Pi_{i}\left(v_{i}\right)=\pi_{i}\left(v_{i}, v_{i}\right)$, equals

$$
\Pi_{i}\left(v_{i}\right)=\Pi_{i}(\underline{v})+\int_{\underline{v}_{i}}^{v_{i}} \int u_{i 1}\left(y, s_{i}\right) X_{i}\left(y, s_{i}\right) d G_{i}\left(s_{i}\right) d y .
$$

Proof. See the Appendix. 
$\Pi_{i}$ in equation (18) resembles $\Pi_{i}^{*}$ in equation (10), the profit function in the benchmark case. If we can implement $X_{i}^{*}$ in a two-stage mechanism with $\Pi_{i}(\underline{v})=0$ then $\Pi_{i}\left(v_{i}\right)=\Pi_{i}^{*}\left(v_{i}\right)$ and the seller's revenue is the same as in the benchmark. The following result - which is the main result of the paper - shows that this is indeed the case.

Theorem 1 When the seller can disclose without observing the buyers' shocks, the same outcome can be implemented as in the benchmark case described in Proposition 1.

Proof. Set $X_{i}=X_{i}^{*}$ and $T_{i}$ so that (14) with (12) and (18) hold. (It is easy to see that this can be done.) By Lemma 3 the mechanism is incentive compatible in the second stage after a truthful announcement of $v_{i}$, hence by Lemma 5 equation (16) holds. Suppose that all buyers except $i$ report their types truthfully and consider buyer $i$ with type $v_{i}$ contemplating to misreport to $\hat{v}_{i}<v_{i}$. His payoff is

$$
\pi_{i}\left(v_{i}, \hat{v}_{i}\right)-\pi_{i}\left(v_{i}, v_{i}\right)=\left[\pi_{i}\left(v_{i}, \hat{v}_{i}\right)-\pi_{i}\left(\hat{v}_{i}, \hat{v}_{i}\right)\right]-\left[\pi_{i}\left(v_{i}, v_{i}\right)-\pi_{i}\left(\hat{v}_{i}, \hat{v}_{i}\right)\right]
$$

By (16) and (18) the difference of the two bracketed expression can be written as

$$
\iint_{\hat{v}_{i}}^{v_{i}} u_{i 1}\left(y, s_{i}\right) X_{i}^{*}\left(\hat{v}_{i}, \sigma_{i}\left(y, \hat{v}_{i}, s_{i}\right)\right) d y d G_{i}\left(s_{i}\right)-\iint_{\hat{v}_{i}}^{v_{i}} u_{i 1}\left(y, s_{i}\right) X_{i}^{*}\left(y, s_{i}\right) d y d G_{i}\left(s_{i}\right)
$$

But since for all $y \in\left[\hat{v}_{i}, v_{i}\right]$, by property (iii) of $X_{i}^{*}$ (Corollary 1 ),

$$
X_{i}^{*}\left(\hat{v}_{i}, \sigma_{i}\left(y, \hat{v}_{i}, s_{i}\right)\right) \leq X_{i}^{*}\left(y, s_{i}\right)
$$

the difference in (19), and hence $\pi_{i}\left(v_{i}, \hat{v}_{i}\right)-\pi_{i}\left(v_{i}, v_{i}\right)$, is non-positive. A similar argument can be used to rule out deviation to $\hat{v}_{i}>v_{i}$.

Buyer $i$ 's participation constraint holds in the first stage (when he only knows $v_{i}$ ) because $\Pi_{i}^{*} \geq 0$. His second-stage participation constraint fails if for some $\left(v_{i}, s_{i}\right)$, $T_{i}^{*}\left(v_{i}, s_{i}\right)>X_{i}^{*}\left(v_{i}, s_{i}\right) u_{i}\left(v_{i}, s_{i}\right)$. This is a consequence of the simplification that transfers are made at the end. By asking buyer $i$ to pay $\gamma_{i}\left(v_{i}\right)=\sup _{s_{i}}\left\{X_{i}^{*}\left(v_{i}, s_{i}\right) u_{i}\left(v_{i}, s_{i}\right)-\right.$ $\left.T_{i}^{*}\left(v_{i}, s_{i}\right)\right\}$ in the first round and then $T_{i}^{*}\left(v_{i}, s_{i}\right)-\gamma_{i}\left(v_{i}\right)$ in the second round, the same allocation is implemented and all interim participation constraints hold.

Finally, we point out that the necessary and sufficent condition that guarantees truthful reports in the first stage is that (19) is non-positive.

A key feature of the optimal allocation rule that makes the proof work is that for all $v_{i}, \hat{v}_{i} \in[\underline{v}, \bar{v}]$ and $s_{i}, \hat{s}_{i} \in \mathbb{R}$ such that $v_{i}>\hat{v}_{i}$ and $u_{i}\left(v_{i}, s_{i}\right)=u_{i}\left(\hat{v}_{i}, \hat{s}_{i}\right)$, the allocation 
rule "favors" the pair $\left(v_{i}, s_{i}\right)$, that is, $X_{i}^{*}\left(\hat{v}_{i}, \hat{s}_{i}\right) \leq X_{i}^{*}\left(v_{i}, s_{i}\right)$, as seen in property (iii) in Corollary 1. In words, buyer $i$ with type $v_{i}$ and a given ex-post valuation wins the object more often than he does with a lower type $\hat{v}_{i}$ but the same ex-post valuation.

\subsection{Necessary and Sufficient Conditions}

The assumptions made in Section 2 (in particular, Assumptions 1 and 2) are not necessary for our main result to hold. Below we discuss what are the necessary and sufficient conditions for the allocation $X_{i}^{*}$, defined by (9), to be implementable by an incentive compatible mechanism in both the benchmark and the original models.

The Benchmark Case. - At the end of the proof of Proposition 1, we have concluded that $X_{i}^{*}$ can be implemented in the benchmark case if and only if for all $i, \widehat{v}_{i}, v_{i} \in[0,1]$,

$$
\iint_{\hat{v}_{i}}^{v_{i}} u_{i 1}\left(y, s_{i}\right) X_{i}^{*}\left(y, s_{i}\right) d y d G_{i}\left(s_{i}\right) \geq \int\left(u_{i}\left(v_{i}, s_{i}\right)-u_{i}\left(\hat{v}_{i}, s_{i}\right)\right) X_{i}^{*}\left(\hat{v}_{i}, s_{i}\right) d G_{i}\left(s_{i}\right),
$$

where $\int_{\widehat{v}_{i}}^{v_{i}}$ denotes $-\int_{v_{i}}^{\widehat{v}_{i}}$ for $v_{i}<\hat{v}_{i}$. Notice that if there were no shocks $\left(s_{i}\right.$ was zero with probability one), this condition would boil down to the necessary condition identified in the standard optimal auction literature (see Myerson (1981)), which requires a buyer with a higher type to win with a higher probability. That is, $X_{i}^{*}$ has to be increasing in $v_{i}$. Because of the shocks, this condition only has to be satisfied in expectation in the benchmark. Our sufficient conditions imply that $X_{i}^{*}$ is increasing in $v_{i}$, not only in expectation, but for all $s_{i}$. This is because Assumptions 1 and 2 guarantee that buyer $i$ 's shock-adjusted virtual valuation, $W_{i}$, is increasing in $v_{i}$ and therefore $X_{i}^{*}$ is increasing, too. As a matter of fact the monotonicity of $W_{i}$ in $v_{i}$ follows form the concavity of $u_{i}$ in $v_{i}$ which is a much weaker requirement than Assumptions 1 and 2.

The Original Model. - Recall that the allocation rule $X_{i}^{*}$ is implemented in a twostage mechanism. In the first stage, buyers report their types. In the second stage, they learn their shocks, and report them. Hence, there will be two conditions, one for each stage, that are necessary and sufficient for incentive compatibility.

The first condition guarantees that at the second stage buyer $i$ reports $s_{i}$ truthfully, given truthful reports in the first stage. At the end of the proof of Lemma 3 we pointed out that this condition holds, if and only if, for all $i, \widehat{s}_{i}, s_{i}$ and $v_{i}$, such that $\widehat{s}_{i}<s_{i}$,

$$
X_{i}^{*}\left(v_{i}, s_{i}\right) \geq X_{i}^{*}\left(v_{i}, \hat{s}_{i}\right)
$$


By the second stage the seller knows the buyers' types. Hence the problem of inducing them to report their new information is essentially the same as in a standard optimal auction. That is why we get the usual condition: a buyer with a larger shock must win the object with higher probability. Assumptions 1 implies that $W_{i}$ is increasing in $s_{i}$ and hence $X_{i}^{*}$ is increasing too. Notice that in the benchmark case it does not matter whether $X_{i}^{*}$ is monotonic in $s_{i}$. The seller observes the shocks and does not have to elicit this information from the buyers. As a result, if (20) holds but (21) does not, $X_{i}^{*}$ can be implemented in the benchmark but not in the original model.

The second condition guarantees that buyer $i$ reports her type truthfully at the first stage. At the end of the proof of Theorem 1 we showed that this holds if and only if for all $i, \widehat{v}_{i}, v_{i} \in[0,1]$,

$$
\iint_{\hat{v}_{i}}^{v_{i}} u_{i 1}\left(y, s_{i}\right) X_{i}^{*}\left(y, s_{i}\right) d y d G_{i}\left(s_{i}\right) \geq \iint_{\hat{v}_{i}}^{v_{i}} u_{i 1}\left(y, s_{i}\right) X_{i}^{*}\left(\hat{v}_{i}, \sigma_{i}\left(y, \hat{v}_{i}, s_{i}\right)\right) d y d G_{i}\left(s_{i}\right)
$$

This condition is probably the hardest to interpret. Recall that the definition of $\sigma_{i}$ is that $u_{i}\left(\hat{v}_{i}, \sigma_{i}\left(v_{i}, \hat{v}_{i}, s_{i}\right)\right)=u_{i}\left(v_{i}, s_{i}\right)$. If one replaces $\sigma_{i}\left(y, \hat{v}_{i}, s_{i}\right)$ by $s_{i}$ in the righthand side of $(22)$, then this condition would coincide with the necessary and sufficient condition in the benchmark, (20). Unlike in the benchmark, if a buyer misreports his type in the first stage, he can also misreport his shock in the second stage. As we have proved in Lemma 4, he would indeed do so, and instead of $s_{i}$, he would report $\sigma_{i}\left(v_{i}, \hat{v}_{i}, s_{i}\right)$. That is, he would make a report in the second stage such that the expost valuation computed from his reports was his true valuation. This explains the discrepancy between (20) and (22). It is not enough that $X_{i}^{*}$ is increasing in $v_{i}$ in expectation, it must be increasing even under the second-stage deviation strategy of the buyers. Again, Assumption 2 implies that (22) does not only hold in expectation but also pointwise. This is because $X_{i}^{*}$ satisfies property (iii) in Corollary 1.

To summarize: Condition (21) is irrelevant for the benchmark, since the seller observes the shocks and does not have to induce the buyers to report them. Condition (22) is stronger than condition (20), because a deviation in the first stage is linked to a deviation in the second stage, which is not possible in the benchmark. Furthermore, Assumption 1 is sufficient for (21), and Assumption 2 is sufficient for (22). The concavity of $u_{i}$ in $v_{i}$ implies (20), which is a weak consequence of Assumptions 1 and 2.

We want to point out that the conditions (20), (21), and (22) can be stated in 
terms of the joint distribution of $V_{i}, v_{i}$, and $z_{i}$ without introducing the variable $s_{i}$. These conditions involve $u_{i}, u_{i 1}$, and $X_{i}^{*}$, which can all be expressed without $s_{i}$. Recall from the proof of Lemma 2, that $s_{i} \equiv H_{v_{i}}^{-1}\left(V_{i}\right), u_{i}\left(v_{i}, s_{i}\right)=V_{i}$, and $u_{i 1}(v, s)=$ $\left(\partial H_{v_{i}}\left(V_{i}\right) / \partial v_{i}\right) / h_{v_{i}}\left(V_{i}\right)$. Therefore, buyer $i$ 's shock-adjusted virtual valuation, $W_{i}$, is

$$
V_{i}-\frac{1-F_{i}\left(v_{i}\right)}{f_{i}\left(v_{i}\right)} \frac{\partial H_{v_{i}}\left(V_{i}\right) / \partial v_{i}}{h_{v_{i}}\left(V_{i}\right)} .
$$

Since according to the optimal allocation rule the object goes to the buyer with the largest shock-adjusted virtual valuation, $X_{i}^{*}$ can also be defined without the introduction of $s_{i}$. However, as we have seen, $X_{i}^{*}$ and the necessary-sufficient monotonicity conditions can be interpreted more easily with the help of the transformed signal, $s_{i}$.

Below we show, by examples, that sometimes the allocation rule $X_{i}^{*}$ is only implementable in the benchmark case, but not in the original model. These examples also illustrate that Assumptions 1 and 2 are not necessary.

Example 4. Suppose that there is a single buyer, and in the transformed model $u(v, s)=\alpha(v+s)+\beta v s$. In order to guarantee that $u$ is increasing in both arguments, assume $(\alpha, \beta) \in A \equiv\{(a, b): a \geq \max \{0,-b\}\}$ and $v, s \in[0,1]$. Let $F(v)=1-(1-v)^{k}$ with $k \geq 1$, and assume $s$ is uniform. We shall characterize the subsets of $A$ such that $X^{*}$ is implementable in the benchmark case and in the original model, respectively.

The shock-adjusted virtual valuation of the buyer is $W(v, s)=\alpha(v+s)+\beta v s-$ $(1-v)(1+\beta s) / k$. Recall that $X^{*}(v, s)=1$ if $W(v, s) \geq 0$, and zero otherwise. Since $\partial W / \partial v=(\alpha+\beta s)(k+1) / k, W$ is increasing in $v$ when $\alpha \geq \max \{0,-\beta\}$. Therefore condition (20) holds for all $(\alpha, \beta) \in A$ and $X^{*}$ is implementable on the entire set $A$.

Condition (21) is satisfied if and only if $W$ is increasing in $s$. Since $\partial W / \partial s=$ $\alpha+\beta v-(1-v) \beta / k$, this requires $\alpha \geq \max \{\beta / k,-\beta\} .{ }^{19}$ Hence, $\alpha \geq \max \{\beta / k,-\beta\}$ is necessary and sufficient for (21) to hold.

Finally, (22) is satisfied if and only if $X^{*}(v, \sigma(v+\delta, v, s)) \leq X^{*}(v+\delta, s)$ whenever $\delta \geq 0$. This holds if and only if $W(v, \sigma(v+\delta, v, s)) \leq W(v+\delta, s)$. We show that this condition holds for all $(\alpha, \beta) \in A$. In this example,

$$
\sigma(v+\delta, v, s)=\frac{\alpha(\delta+s)+\beta(v+\delta) s}{\alpha+\beta v}
$$

\footnotetext{
${ }^{19}$ If $\alpha<\max \{\beta / k,-\beta\}$, then there exists an interval on which $W$ is decreasing in $s$, which implies that (21) does not hold.
} 
Therefore $W(v+\delta, s)-W(v, \sigma(v+\delta, v, s))$ is

$$
\frac{1-v}{k}\left(\alpha+\beta \frac{\alpha(\delta+s)+\beta(v+\delta) s}{\alpha+\beta v}\right)-\frac{1-v-\delta}{k}(\alpha+\beta s)=\frac{\delta(\alpha+\beta s)}{k} \frac{\alpha+\beta}{\alpha+\beta v}>0 .
$$

We conclude that while $X^{*}$ is implementable in the benchmark case for all $(\alpha, \beta) \in$ $A$, in the original model it is only implementable for $\alpha \geq \max \{\beta / k,-\beta\}$.

This example also shows that Assumptions 1 and 2 are not necessary for our results. Notice that Assumption 1 holds if and only if $\beta \leq 0$, and Assumption 2 holds if and only if $\beta \geq 0$. However $X^{*}$ is implementable whenever $\alpha \geq \max \{\beta / k,-\beta\}$.

In the following two examples the orthogonal transformation yields special cases of the parametric model of Example 4.

Example 5. Suppose that there is a single buyer whose true valuation is distributed according to the cdf $V^{2}$ on $[0,1]$. His signal, $v$, is a uniform draw from $[0, V]$. Assume that the seller can disclose, without observing, the realization of $V$ to the buyer.

Notice that the joint distribution of $(V, v)$ is uniform on the triangle $0 \leq v \leq V \leq 1$. As a result, the conditional distribution of $V$ given $v$ is also uniform on the interval $[v, 1]$ with cdf $H_{v}(V)=(V-v) /(1-v)$. The unconditional cdf of $v$ is $F(v)=1-(1-v)^{2}$. To perform the transformation, define $s \equiv H_{v}(V)$. Then, $u(v, s)=H_{v}^{-1}(s)=v+s-v s$.

By direct calculation, $u_{1}=1-s, u_{11}=0, u_{2}=1-v, u_{12}=-1$. Therefore Assumption 1 holds $\left(u_{12} \leq 0\right)$, but Assumption 2 does not $\left(u_{11} / u_{1}>u_{12} / u_{2}\right)$. However, the model is equivalent to Example 4 with $k=2, \alpha=1, \beta=-1$. Since $\alpha \geq \max \{\beta / k,-\beta\}$, $X^{*}$ can be implemented in the original model. This illustrates that Assumptions 1 and 2 are not necessary for Theorem 1.

It is interesting to note that the shock-adjusted virtual valuation simplifies to $W=$ $[3 u(v, s)-1] / 2$. Recall that in the optimal allocation the buyer gets the good whenever $W \geq 0$, that is, $u(v, s) \equiv V \geq 1 / 3$. The allocation rule only depends on the buyer's true valuation, and not on his original signal, $v$. In this example (and its extension to multiple symmetric buyers), there is no distortion in the allocation of the good conditional on sale.

Example 6. Suppose that the joint pdf of $v$ and $V$ is $\phi(v, V)=1 / v$ for $0 \leq V \leq v \leq 1$, and the seller can disclose $V$ to the buyer. The pdf of $v$ is $f(v) \equiv \phi_{v}(v)=\int_{0}^{v} 1 / v d V=1$, i.e., uniform on $[0,1]$. The distribution of $V$ conditional on $v$ is $H_{v}(V)=V / v$, i.e., uniform on $[0, v]$. Define $s \equiv H_{v}(V)=V / v$. Then, $u(v, s)=H_{v}^{-1}(s)=v s$. 
This model is equivalent to Example 4 with $k=1, \alpha=0, \beta=1$. Since $\max \{\beta / k,-\beta\}>$ $\alpha \geq \max \{0,-\beta\}, X^{*}$ cannot be implemented in the original model. $\square$

\subsection{Discussion of the Optimal Mechanism}

As we have pointed out, in the optimal mechanism, two ex-ante symmetric buyers with the same ex-post valuation may not have the same probability of winning. According to Corollary 1 , an increase in a buyer's type, holding his true valuation constant, weakly increases the probability that he wins the object. ${ }^{20}$ This is so because $W_{i}\left(v_{i}, s_{i}\right)$ in equation (8) is weakly increasing in $v_{i}$ even if $u_{i}\left(v_{i}, s_{i}\right)$ is compensated. (Example 5 is a knife-edge case where $W_{i}$ remains constant for such a compensated change.) The economic reason for this property is that the seller uses discrimination-giving preference to someone with a higher type -in order to screen the buyers.

This property implies that the auction is not efficient, even under ex-ante symmetry of the bidders and conditional on the object being sold. In contrast, in the classical symmetric setup with deterministic valuations, the optimal auction of Myerson (1981) and Riley and Samuelson (1981) is efficient conditional on sale.

In order to better explain Theorem 1, consider a setup where the buyers are ex ante symmetric (the $v_{i}$ 's are identically distributed), and the shocks are additive, mean zero random variables. Let us compare the optimal allocation rule in our model with that of the revenue maximizing auction when nobody (neither the seller nor the buyers) can observe them. In the latter case, the seller should allocate the good to the buyer with the largest non-negative virtual value-estimate, $v_{i}-\left(1-F\left(v_{i}\right)\right) / f\left(v_{i}\right)$. In our model, in the optimal mechanism, the good will be allocated more efficiently, as the winner will now be the buyer with the highest non-negative shock-adjusted virtual valuation, $v_{i}+s_{i}-\left(1-F\left(v_{i}\right)\right) / f\left(v_{i}\right){ }^{21}$ According to Theorem 1, the seller, by controlling the release of the shocks and without actually observing them, can implement the this rule and appropriate the increase in efficiency. ${ }^{22}$

\footnotetext{
${ }^{20}$ In the previous section, we showed that this is a necessary condition for implementation when the seller cannot observe her signals. However, it is not necessary in the benchmark, when the seller can observe the shocks.

${ }^{21}$ It is easy to see that if $v_{i}-\left(1-F\left(v_{i}\right)\right) / f\left(v_{i}\right)<v_{j}-\left(1-F\left(v_{j}\right)\right) / f\left(v_{j}\right)$, but, by adding the shocks to both sides the inequality is reversed, then $v_{i}+s_{i}>v_{j}+s_{j}$. Therefore, an allocation based on the shock-adjusted virtual valuations "pointwise" improves efficiency. (This may not be true if the $F_{i}$ 's are not identical.)

${ }^{22}$ If the buyers' ex ante type-distributions are not identical then, as the seller gets to observe the
} 


\section{The Handicap Auction}

In this section we show how to implement the optimal mechanism when in the transformed model $u_{i 1}$ is constant, like in Examples 1 and 2a. We call the mechanism handicap auction.

A handicap auction consists of two rounds. In the first round, each buyer $i$, knowing his type, chooses a price premium $p_{i}$ for a fee $C_{i}\left(p_{i}\right)$, where $C_{i}$ is a fee-schedule published by the seller. The buyers do not observe the premia chosen by others. The second round is a traditional auction, but the winner is required to pay his premium over the price. Between the two rounds, the seller may send messages to the buyers. In the optimal handicap auction the seller discloses her signals to the buyers, and the second round is a second-price (or English) auction with a zero reservation price. We call this mechanism a handicap auction because in the second round, the buyers compete under unequal conditions: a bidder with a smaller premium has an advantage. The bidders buy their premium in the initial round, which is a form of price discrimination. ${ }^{23}$ If there is only a single buyer, then, as we have seen it in Section 3, the handicap auction simplifies to a menu of buy options: $p_{i}$ can be thought of as the strike price, and the upfront fee, $C_{i}\left(p_{i}\right)$, is the cost of the option.

It is a dominant strategy for buyer $i$ who chose premium $p_{i}$ in the first stage to bid $u_{i}\left(v_{i}, s_{i}\right)-p_{i}$ in the second stage of the handicap auction. Assuming that the buyers follow this weakly dominant strategy in the second round, the handicap auction can be represented by pairs of functions, $p_{i}:[\underline{v}, \bar{v}] \rightarrow \mathbb{R}$ and $c_{i}:[\underline{v}, \bar{v}] \rightarrow \mathbb{R}$, for $i=1, \ldots, n$, where $p_{i}\left(v_{i}\right)$ is the price premium that in equilibrium type $v_{i}$ picks for a fee of $c_{i}\left(v_{i}\right) \equiv C_{i}\left(p_{i}\left(v_{i}\right)\right)$.

Proposition 2 If $u_{i 1}$ is constant then the optimal mechanism of Theorem 1 can be implemented via a handicap auction $\left\{c_{i}, p_{i}\right\}_{i=1}^{n}$, where

$$
p_{i}\left(v_{i}\right)=\frac{1-F_{i}\left(v_{i}\right)}{f_{i}\left(v_{i}\right)} u_{i 1},
$$

signals, the efficiency of the optimal mechanism may only improve in ex ante expectation. Still, there will be some efficiency gain, which will be extracted by the seller even if she cannot observe the additional signals.

${ }^{23}$ The handicap auction can also be implemented as a mechanism where, in the first round, each bidder buys a discount (larger discounts cost more), and then participates in a second price auction with a positive reservation price, where the winner's discount is applied towards his payment. 
and $c_{i}\left(v_{i}\right)$ is defined by

$c_{i}\left(v_{i}\right)=\iint\left[\max \left\{W_{i}\left(v_{i}, s_{i}\right)-W_{-i}^{0}\left(v_{-i}, s_{-i}\right), 0\right\}-\int_{\underline{v}}^{v_{i}} u_{i 1} \mathbf{1}_{\left\{W_{i}\left(y, s_{i}\right) \geq W_{-i}^{0}\left(v_{-i}, s_{-i}\right)\right\}} d y\right] d F_{-i} d G$,

where $W_{i}\left(v_{i}, s_{i}\right)=u_{i}\left(v_{i}, s_{i}\right)-p_{i}\left(v_{i}\right)$ and $W_{-i}^{0}\left(v_{-i}, s_{-i}\right)=\max _{j \neq i}\left\{W_{j}\left(v_{j}, s_{j}\right), 0\right\}$.

From the seller's perspective, the premium-fee schedule works as a device to discriminate among buyers with different value estimates. A buyer with a high type, expecting to win often, chooses a small price premium for a large fee in order not to pay much when he wins. Low types choose large price premia, which are cheaper, but make winning more expensive. In the optimal handicap auction, just like in the general optimal mechanism, two buyers with the same ex-post valuation do not have the same probability of winning. The buyer with a larger $v_{i}$ chooses a smaller price premium, bids higher in the second round, and ends up winning more often.

One may suggest that the way the seller can appropriate all rents from the additional information is that in the handicap auction, she essentially charges the buyers a typedependent up-front fee equal to the "value" of the information they are about to receive. This intuition may be appealing, but it overly simplifies the workings of the mechanism. The value of the additional information to the participants is not well-defined because it depends on the rules of the selling mechanism. This value could be different if the seller chose a mechanism different from the handicap auction.

It may be useful to compute a numerical example not only for illustrative purposes, but also, to see how a seller may be able to calculate the parameters of the optimal handicap auction (the price premium-fee schedule) in a practical application.

Example 1, revisited. Consider the setup of Example 1, with the following additional assumptions: The buyers' types are distributed uniformly on $[0,1]$, and the shocks are distributed according to a standard logistic distribution. ${ }^{24}$

First, suppose that there is a single buyer. Then, as seen also in Section 3, the handicap auction can be thought of as a menu of buy options, represented by $C_{1}\left(p_{1}\right)$, where $p_{1}$ is the strike price and $C_{1}\left(p_{1}\right)$ is the fee of the option. In the first round, the buyer chooses a price $p_{1}$ and pays $C_{1}\left(p_{1}\right)$; in the second round (after having observed $s_{1}$ ), he has the option to buy the good at price $p_{1}$. Again, we represent this menu as a

\footnotetext{
${ }^{24}$ The cdf of the standard logistic distribution is $G_{i}\left(s_{i}\right)=e^{s_{i}} /\left(1+e^{s_{i}}\right), s_{i} \in(-\infty,+\infty)$.
} 
pair of functions, $c_{1}\left(v_{1}\right)$ and $p_{1}\left(v_{1}\right), v_{1} \in[0,1]$.

The expected revenue maximizing strike price-schedule is given by $(23), p_{1}\left(v_{1}\right)=$ $1-v_{1}$. The fee-schedule in (24) becomes,

$$
c_{1}\left(v_{1}\right)=\frac{1}{2} \ln (1+e)-1+v_{1}+\frac{1}{2} \ln \left(1+e^{1-2 v_{1}}\right) .
$$

We can also express the cost of the option as a function of the strike price,

$$
c_{1}=C_{1}\left(p_{1}\right)=\frac{1}{2} \ln \left[(1+e)\left(1+e^{2 p_{1}-1}\right)\right]-p_{1} .
$$

$C_{1}$ is a strictly decreasing function on $[0, \infty)$. The buyer with a higher estimate buys an option with a lower strike price at a higher cost. For example, if the buyer has the lowest estimate, $v_{1}=0$, then he buys the option of getting the good at $p_{1}=1$, which $\operatorname{costs} c_{1}=\ln [(1+e) / e] \approx 0.31$ upfront, and yields zero net surplus. In contrast, the highest type, $v_{1}=1$, buys a call option with zero strike price at a cost of about 0.81 .

As the number of buyers increases, the premium-fee schedule, $C_{i}\left(p_{i}\right)$, shifts down and flattens out. This is so because the premium as a function of the buyer's type, given by (23), remains the same, $p_{i}\left(v_{i}\right)=1-v_{i}$. As there are more buyers in the second round of the auction, competition increases and the bidders' gains from learning their valuations precisely decreases. As a result, they are willing to pay less up front: the fee schedule, $c_{i}\left(v_{i}\right)$, computed using (24), decreases for all $v_{i}$ as $n$ increases.

\section{Conclusions}

We have analyzed an auction model where the seller can reveal without observing signals to the buyers that, together with the buyers' original private information, affect their valuations. We have derived the expected revenue maximizing mechanism comprising both the rules of information disclosure and those of the transaction.

In the optimal mechanism, the seller discloses all the information that she can and obtains the same expected revenue as if she could observe the "new" part of the additional signals that she can release without observing. The buyers do not enjoy any additional information rents from the signals whose disclosure is controlled by the seller. We have provided necessary and sufficient conditions for this result. The optimal mechanism can be implemented via a "handicap auction" in interesting applications. 
There are selling mechanisms that have similar features to our optimal auction. For a single buyer, the optimal mechanism is a menu of "buy" options, where the cost of the option is negatively related to the strike price of the good. Similar contracts are used in financial, labor, and other production relationships. Sellers often demand that bids incorporate payments contingent on further exploration of the good's value, just like in our optimal mechanism. ${ }^{25}$ Firms that solicit bids from underwriters before their IPO's favor the most aggressive bidders in subsequent bidding for financial contracts. ${ }^{26}$ This type of mechanisms, like our optimal auction, has the feature that ex-post discrimination among bidders is based upon a prior contractual relationship.

The overall conclusion of our investigation is that under certain conditions, the seller who controls the "flow of information" in an auction appropriates the rents of that information.

\section{Appendix: Omitted Proofs}

Proof of Lemma 1. (Monotonicity) Differentiating the identity $s_{i} \equiv H_{i v_{i}}\left(u_{i}\left(v_{i}, s_{i}\right)\right.$ ) in $v_{i}$ and $s_{i}$ respectively, rearranging, and using $u_{i}\left(v_{i}, s_{i}\right)=V_{i}$ yield,

$$
\begin{aligned}
& \frac{\partial u_{i}\left(v_{i}, s_{i}\right)}{\partial v_{i}}=-\frac{\partial H_{i v_{i}}\left(V_{i}\right) / \partial v_{i}}{h_{i v_{i}}\left(V_{i}\right)} \\
& \frac{\partial u_{i}\left(v_{i}, s_{i}\right)}{\partial s_{i}}=\frac{1}{h_{i v_{i}}\left(V_{i}\right)}
\end{aligned}
$$

The density $h_{i v_{i}}$ is positive, and by assumption $\partial H_{i v_{i}}\left(V_{i}\right) / \partial v_{i}<0$, therefore $u_{i}$ is strictly increasing in both arguments.

(ii) Suppose that $s_{i}$ and $\tilde{s}_{i}$ are independent of $v_{i}$, and there exist functions $u_{i}$ and $\tilde{u}_{i}$, strictly increasing in their second argument, such that $u_{i}\left(v_{i}, s_{i}\right) \equiv \tilde{u}_{i}\left(v_{i}, \tilde{s}_{i}\right) \equiv V_{i}{ }^{27}$ We want to show that $\tilde{s}_{i}$ is a monotonic transformation of $s_{i}$, that is, there exists an increasing function $\lambda$ such that for all $v_{i}$ and $\sigma$,

$$
\operatorname{Pr}\left(u_{i}\left(v_{i}, s_{i}\right) \leq u_{i}\left(v_{i}, \sigma\right)\right)=\operatorname{Pr}\left(\tilde{u}_{i}\left(v_{i}, \tilde{s}_{i}\right) \leq \tilde{u}_{i}\left(v_{i}, \lambda(\sigma)\right)\right)
$$

\footnotetext{
${ }^{25}$ See, for example, the privatization of the Antamina mine by the Peruvian government, as described in Moel and Tufano (1997).

${ }^{26}$ See Krigman et al. (2001) and the references therein.

${ }^{27}$ The latter condition means that for all $\xi \in \mathbb{R}, \operatorname{Pr}\left(u_{i}\left(v_{i}, s_{i}\right) \leq \xi\right)=H_{i v_{i}}(\nu)=\operatorname{Pr}\left(\tilde{u}_{i}\left(v_{i}, \tilde{s}_{i}\right) \leq \xi\right)$.
} 
The issue is that $\lambda$ must be the same function for all realizations of $v_{i}$.

Define $\lambda$ so that for all $\sigma, \operatorname{Pr}\left(s_{i} \leq \sigma\right)=\operatorname{Pr}\left(\tilde{s}_{i} \leq \lambda(\sigma)\right)$. (The function $\lambda$ associates the same percentiles of $s_{i}$ and $\tilde{s}_{i}$ with each other.) Note that this function is increasing and does not depend on the realization of $v_{i}$ as both $s_{i}$ and $\tilde{s}_{i}$ are independent of $v_{i}$. By the monotonicity of $u_{i}$ in its second argument, the lefthand side of $(27)$ equals $\operatorname{Pr}\left(s_{i} \leq \sigma\right)$, which in turn equals $\operatorname{Pr}\left(\tilde{s}_{i} \leq \lambda(\sigma)\right)$ by the definition of $\lambda$. By the monotonicity of $\tilde{u}_{i}$ in its second argument, $\operatorname{Pr}\left(\tilde{s}_{i} \leq \lambda(\sigma)\right)$ equals $\operatorname{Pr}\left(\tilde{u}_{i}\left(v_{i}, \tilde{s}_{i}\right) \leq \tilde{u}_{i}\left(v_{i}, \lambda(\sigma)\right)\right)$, which is the right-hand side of $(27)$, therefore the equality indeed holds.

Proof of Lemma 2. In this proof we omit the reference to the identity of buyer $i$. Recall from Lemma 1 that the model can be transformed into one with orthogonally normalized $v$ and $s$ by letting $s \equiv H_{v}(V)$ and $u(v, s)=H_{v}^{-1}(s)$. First, we prove the lemma for this $s$ and $u$, and then argue that the choice of $s$ and $u$ does not matter.

By partially differentiating $\left(\partial H_{i v_{i}}\left(V_{i}\right) / \partial v_{i}\right) / h_{i v_{i}}\left(V_{i}\right)$ Assumption 1 can be written as

$$
\frac{\partial h_{v} / \partial v}{\partial H_{v} / \partial v} \leq \frac{h_{v}^{\prime}}{h_{v}}
$$

and Assumption 2 can be expressed as

$$
\frac{\partial^{2} H_{v}(V)}{\partial v^{2}} / \frac{\partial H_{v}(V)}{\partial v} \leq \frac{\partial h_{v}(V)}{\partial v} / h_{v}(V)
$$

From (26), using the chain rule we get,

$$
\begin{aligned}
\frac{\partial^{2} u(v, s)}{\partial s \partial v} & \left.=-\frac{1}{h_{v}^{2}(V)}\left(\frac{\partial h_{v}(V)}{\partial v}+h_{v}^{\prime}(V) \frac{\partial H_{v}^{-1}(s)}{\partial v}\right)\right\rfloor_{V=H_{v}^{-1}(s)} \\
. & \left.=\frac{1}{h_{v}^{2}(V)}\left(\frac{h_{v}^{\prime}(V)}{h_{v}(V)} \frac{\partial H_{v}(V)}{\partial v}-\frac{\partial h_{v}(V)}{\partial v}\right)\right\rfloor_{V=H_{v}^{-1}(s)}
\end{aligned}
$$

where the second line follows from (25). Since the density $h_{v}$ is positive, and $\partial H_{v} / \partial v \leq$ $0, u_{12} \leq 0$ translates into $(28)$.

Differentiate (25) in $v$ to get

$$
\frac{\partial^{2} u(v, s)}{\partial v^{2}}=-\frac{\partial\left[\partial H_{v}\left(H_{v}^{-1}(s)\right) / \partial v\right] / h_{v}\left(H_{v}^{-1}(s)\right)}{\partial v}
$$


This can be rewritten as

$$
\begin{aligned}
\frac{\partial^{2} u(v, s)}{\partial v^{2}} & =-\frac{1}{h_{v}(V)}\left[\frac{\partial^{2} H_{v}(V)}{\partial v^{2}}-\frac{\partial H_{v}(V)}{\partial v} \frac{\partial h_{v}(V)}{\partial v} \frac{1}{h_{v}(V)}\right] \\
& +\frac{1}{h_{v}^{2}(V)} \frac{\partial H_{v}(V)}{\partial v}\left[\frac{\partial h_{v}(V)}{\partial v}-\frac{h_{v}^{\prime}(V)}{h_{v}(V)} \frac{\partial H_{v}(V)}{\partial v}\right] \\
& =\frac{1}{h_{v}(V)}\left[\frac{2}{h_{v}(V)} \frac{\partial H_{v}(V)}{\partial v} \frac{\partial h_{v}(V)}{\partial v}-\frac{h_{v}^{\prime}(V)}{h_{v}^{2}(V)}\left(\frac{\partial H_{v}(V)}{\partial v}\right)^{2}-\frac{\partial^{2} H_{v}(V)}{\partial v^{2}}\right],
\end{aligned}
$$

where $V=H_{v}^{-1}(s)$. Hence, using this expression and (25),

$\left.\frac{\partial^{2} u(v, s)}{\partial v^{2}} / \frac{\partial u(v, s)}{\partial v}=\frac{-2}{h_{v}(V)} \frac{\partial h_{v}(V)}{\partial v}+\frac{h_{v}^{\prime}(V)}{h_{v}^{2}(V)} \frac{\partial H_{v}(V)}{\partial v}+\frac{\partial^{2} H_{v}(V)}{\partial v^{2}} / \frac{\partial H_{v}(V)}{\partial v}\right\rfloor_{V=H_{v}^{-1}(s)}$.

By (30) and (26),

$$
\left.\frac{\partial^{2} u(v, s)}{\partial v \partial s} / \frac{\partial u(v, s)}{\partial s}=\frac{1}{h_{v}(V)}\left(\frac{h_{v}^{\prime}(V)}{h_{v}(V)} \frac{\partial H_{v}(V)}{\partial v}-\frac{\partial h_{v}(V)}{\partial v}\right)\right\rfloor_{V=H_{v}^{-1}(s)} .
$$

Hence $u_{i 11} / u_{i 1} \leq u_{i 12} / u_{i 2}$ is equivalent to

$$
\begin{aligned}
& \frac{h_{v}^{\prime}(V)}{h_{v}^{2}(V)} \frac{\partial H_{v}(V)}{\partial v}+\frac{\partial^{2} H_{v}(V)}{\partial v^{2}} / \frac{\partial H_{v}(V)}{\partial v}-\frac{2}{h_{v}(V)} \frac{\partial h_{v}(V)}{\partial v} \\
& \leq \frac{h_{v}^{\prime}(V)}{h_{v}^{2}(V)} \frac{\partial H_{v}(V)}{\partial v}-\frac{1}{h_{v}(V)} \frac{\partial h_{v}(V)}{\partial v} .
\end{aligned}
$$

The first terms in each side cancel, and we get exactly (29).

So far we assumed that $u(v, s)=H_{v}^{-1}(s)$. It remains to show that the choice of $s_{i}$ and $u_{i}$ do not affect the statement of this lemma as long as they satisfy part (i) in Lemma 1. To see this, consider a positive monotonic transformation of $s_{i}, \tilde{s}_{i} \equiv \lambda\left(s_{i}\right)$, and a corresponding $\tilde{u}_{i}$ with $\tilde{u}_{i}\left(v_{i}, \tilde{s}_{i}\right) \equiv u_{i}\left(v_{i}, \lambda\left(s_{i}\right)\right)$. For simplicity assume $\lambda$ is differentiable, hence $\lambda^{\prime}>0$. Since $\tilde{u}_{i 1}=u_{i 1}, \tilde{u}_{i 2}=u_{i 2} \lambda^{\prime}, \tilde{u}_{i 11}=u_{i 11}$, and $\tilde{u}_{i 12}=u_{i 12} \lambda^{\prime}$, the statement of this lemma is valid for $\tilde{u}_{i}$ whenever it is valid for $u_{i}$.

Proof of Proposition 1. Fix a direct mechanism and define

$$
X_{i}\left(v_{i}, s_{i}\right)=\iint x_{i}\left(v_{i}, v_{-i}, s_{i}, s_{-i}\right) d F_{-i}\left(v_{-i}\right) d G_{-i}\left(s_{-i}\right)
$$


If buyer $i$ with type $v_{i}$ reports type $\hat{v}_{i}$ then his profit is,

$$
\pi_{i}\left(v_{i}, \hat{v}_{i}\right)=\iint\left[x_{i}\left(\hat{v}_{i}, v_{-i}, s_{i}, s_{-i}\right) u_{i}\left(v_{i}, s_{i}\right)-t_{i}\left(\hat{v}_{i}, v_{-i}, s_{i}, s_{-i}\right)\right] d F_{-i}\left(v_{-i}\right) d G(s) .
$$

The mechanism is incentive compatible if and only if $\pi_{i}\left(v_{i}, \hat{v}_{i}\right) \leq \pi_{i}\left(v_{i}, v_{i}\right)$, for all $i$ and all $v_{i}, \hat{v}_{i} \in[\underline{v}, \bar{v}]$. Define $\Pi_{i}\left(v_{i}\right) \equiv \pi_{i}\left(v_{i}, v_{i}\right)$ as the equilibrium (or indirect) profit function of buyer $i$. The seller's expected revenue can be written as the difference of the social surplus and the buyers' expected profits,

$$
\sum_{i=1}^{n} \iint\left[u_{i}\left(v_{i}, s_{i}\right) x_{i}\left(v_{i}, v_{-i}, s_{i}, s_{-i}\right)-\Pi_{i}\left(v_{i}\right)\right] d F(v) d G(s) .
$$

The benchmark problem is to maximize (33) subject to the buyers' incentive compatibility and participation constraints.

The incentive constraint guaranteeing that a bidder with type $v_{i}$ does not report $\hat{v}_{i}$ (and vice versa) can be rewritten as

$$
\begin{aligned}
\int\left[u_{i}\left(v_{i}, s_{i}\right)-u_{i}\left(\hat{v}_{i}, s_{i}\right)\right] X_{i}\left(\hat{v}_{i}, s_{i}\right) d G_{i}\left(s_{i}\right) & \leq \pi_{i}\left(v_{i}, v_{i}\right)-\pi_{i}\left(\hat{v}_{i}, \hat{v}_{i}\right) \\
& \leq \int\left[u_{i}\left(v_{i}, s_{i}\right)-u_{i}\left(\hat{v}_{i}, s_{i}\right)\right] X_{i}\left(v_{i}, s_{i}\right) d G_{i}\left(s_{i}\right)
\end{aligned}
$$

From this inequality chain, using the standard techniques of Bayesian mechanism design, one can conclude that

$$
\Pi_{i}\left(v_{i}\right)=\Pi_{i}\left(\underline{v_{i}}\right)+\int_{\underline{v_{i}}}^{v_{i}} \int_{s_{i}} u_{i 1}\left(y, s_{i}\right) X_{i}\left(y, s_{i}\right) d G_{i}\left(s_{i}\right) d y .
$$

Given the profit function of buyer type $v_{i}$ in (35), we can write the seller's expected revenue (after the application of Fubini's Theorem) as

$$
\iint \sum_{i} x_{i}\left(v_{i}, v_{-i}, s_{i}, s_{-i}\right)\left[u_{i}\left(v_{i}, s_{i}\right)-\frac{1-F_{i}\left(v_{i}\right)}{f_{i}\left(v_{i}\right)} u_{i 1}\left(v_{i}, s_{i}\right)\right] d F(v) d G(s) .
$$

The allocation rule $x_{i}^{*}\left(v_{i}, v_{-i}, s_{i}, s_{-i}\right)$ maximizes the integrand in (36) pointwise.

We need to show that this rule is incentive compatible. The candidate optimal allocation rule, $x_{i}^{*}\left(v_{i}, v_{-i}, s_{i}, s_{-i}\right)$, hence $X_{i}^{*}\left(v_{i}, s_{i}\right)$, is weakly increasing in $v_{i}$. This is because $W_{i}$ is increasing in $v_{i}$ since, by assumption, $u_{i}$ is increasing in $v_{i},\left(1-F_{i}\right) / f_{i}$ is 
weakly decreasing in $v_{i}$, and finally, Assumptions 1 and 2 imply that $u_{i}$ is concave in $v_{i}$. Suppose $v_{i}>\hat{v}_{i}$ and use (35) to rewrite

$$
\Pi_{i}^{*}\left(v_{i}\right)-\Pi_{i}^{*}\left(\hat{v}_{i}\right)=\iint_{\hat{v}_{i}}^{v_{i}} u_{i 1}\left(y, s_{i}\right) X_{i}^{*}\left(y, s_{i}\right) d y d G_{i}\left(s_{i}\right)
$$

Since $X_{i}^{*}\left(y, s_{i}\right)$ is increasing in $y$,

$$
\begin{aligned}
\iint_{\hat{v}_{i}}^{v_{i}} u_{i 1}\left(y, s_{i}\right) X_{i}^{*}\left(y, s_{i}\right) d y d G_{i}\left(s_{i}\right) & \geq \iint_{\hat{v}_{i}}^{v_{i}} u_{i 1}\left(y, s_{i}\right) d y X_{i}^{*}\left(\hat{v}_{i}, s_{i}\right) d G_{i}\left(s_{i}\right) \\
& =\int\left(u_{i}\left(v_{i}, s_{i}\right)-u_{i}\left(\hat{v}_{i}, s_{i}\right)\right) X_{i}^{*}\left(\hat{v}_{i}, s_{i}\right) d G_{i}\left(s_{i}\right) .
\end{aligned}
$$

But this is exactly the incentive compatibility condition for buyer type $v_{i}$ not to imitate $\hat{v}_{i}<v_{i},(34)$. A similar argument applies when $\hat{v}_{i}>v_{i}$.

We want to point out that the necessary and sufficient condition for $X_{i}^{*}$ to be implementable is (37), where, by convention, if $v_{i}<\widehat{v}_{i}, \int_{\widehat{v}_{i}}^{v_{i}}=-\int_{v_{i}}^{\widehat{v}_{i}}$. This condition may hold even if $X_{i}^{*}$ is not increasing in its first argument.

Proof of Corollary 1. Continuity of $X_{i}^{*}$ follows because the distributions are atomless. $X_{i}^{*}$ is weakly increasing in $v_{i}$ and $s_{i}$ because (8) is strictly increasing in both variables, which in turn follows from the monotone hazard rate condition, $u_{i 1}>0, u_{i 11} \leq 0$, $u_{i 2}>0$, and $u_{i 12} \leq 0$. Finally, (iii) is another monotonicity property stating that $X_{i}^{*}$ is weakly increasing in $v_{i}$ even if $s_{i}$ is adjusted to keep $u_{i}\left(v_{i}, s_{i}\right)$ constant. To see this, consider $v_{i}>\hat{v}_{i}$ and $s_{i}<\hat{s}_{i}$ with $u_{i}\left(v_{i}, s_{i}\right)=u_{i}\left(\hat{v}_{i}, \hat{s}_{i}\right)$, and compare (8) for $\left(v_{i}, s_{i}\right)$ and $\left(\hat{v}_{i}, \hat{s}_{i}\right)$. The first term of $(8)$ is the same by assumption; in the second term $\left(1-F_{i}\left(v_{i}\right)\right) / f_{i}\left(v_{i}\right) \leq\left(1-F_{i}\left(\hat{v}_{i}\right)\right) / f_{i}\left(\hat{v}_{i}\right)$ by the monotone hazard rate condition, $u_{i 1}\left(v_{i}, s_{i}\right) \leq u_{i 1}\left(\hat{v}_{i}, \hat{s}_{i}\right)$ because $u_{i 11} / u_{i 1} \leq u_{i 12} / u_{i 2}$ (see Footnote 12).

Proof of Lemma 3. Rewrite (13) as

$$
\tilde{\pi}_{i}\left(s_{i}, \hat{s}_{i} ; v_{i}\right)=\tilde{\pi}_{i}\left(\hat{s}_{i}, \hat{s}_{i} ; v_{i}\right)+\left[u_{i}\left(v_{i}, s_{i}\right)-u_{i}\left(v_{i}, \hat{s}_{i}\right)\right] X_{i}\left(v_{i}, \hat{s}_{i}\right)
$$

By reversing the roles of $s_{i}$ and $\hat{s}_{i}$, we get

$$
\tilde{\pi}_{i}\left(\hat{s}_{i}, s_{i} ; v_{i}\right)=\tilde{\pi}_{i}\left(s_{i}, s_{i} ; v_{i}\right)-\left[u_{i}\left(v_{i}, s_{i}\right)-u_{i}\left(v_{i}, \hat{s}_{i}\right)\right] X_{i}\left(v_{i}, s_{i}\right) .
$$

From these equalities standard techniques of Bayesian mechanism design yield (14). 
To see the second part of the claim, suppose buyer $i$ reports $\hat{s}_{i}<s_{i}$ after seeing $s_{i}$. His deviation gain is

$$
\tilde{\pi}_{i}\left(s_{i}, \hat{s}_{i} ; v_{i}\right)-\tilde{\pi}_{i}\left(s_{i}, s_{i} ; v_{i}\right)=\left[\tilde{\pi}_{i}\left(s_{i}, \hat{s}_{i} ; v_{i}\right)-\tilde{\pi}_{i}\left(\hat{s}_{i}, \hat{s}_{i} ; v_{i}\right)\right]-\left[\tilde{\pi}_{i}\left(s_{i}, s_{i} ; v_{i}\right)-\tilde{\pi}_{i}\left(\hat{s}_{i}, \hat{s}_{i} ; v_{i}\right)\right] .
$$

Recall from (38) that the first bracketed difference is $\left[u_{i}\left(v_{i}, s_{i}\right)-u_{i}\left(v_{i}, \hat{s}_{i}\right)\right] X_{i}\left(v_{i}, \hat{s}_{i}\right)$, and by (14) the second one can be rewritten as

$$
\int_{\hat{s}_{i}}^{s_{i}} u_{i 2}\left(v_{i}, z\right) X_{i}\left(v_{i}, z\right) d z \geq \int_{\hat{s}_{i}}^{s_{i}} u_{i 2}\left(v_{i}, z\right) X_{i}\left(v_{i}, \hat{s}_{i}\right) d z=\left[u_{i}\left(v_{i}, s_{i}\right)-u_{i}\left(v_{i}, \hat{s}_{i}\right)\right] X_{i}\left(v_{i}, \hat{s}_{i}\right) .
$$

The inequality follows from $X_{i}$ being weakly increasing in $s_{i}$. Therefore, the gain from deviation is non-positive. A similar argument can be used to show that a deviation to $\hat{s}_{i}>s_{i}$ is not profitable either. We want to point out that (39) is the necesarry and sufficient condition that guarantees that bidder $i$ truthfully reports her shock, given truthful reports in the first stage. Also notice that if the allocation $X_{i}$ is continuously differentiable in its second argument $X_{i}$ being weakly increasing in $s_{i}$ is equivalent to (39). (This is because if $X_{i}$ was not increasing in $s_{i}$, there would exists an interval $\left[\hat{s}_{i}, s_{i}\right]$, where $X_{i}$ is increasing, and hence the oppositite inequality would hold.)

Proof of Lemma 5. Buyer $i$ 's expected profit when his type is $v_{i}$ but reports $\hat{v}_{i}$ in the first round is,

$$
\pi_{i}\left(v_{i}, \hat{v}_{i}\right)=\int\left[u_{i}\left(v_{i}, s_{i}\right) X_{i}\left(\hat{v}_{i}, \sigma_{i}\left(v_{i}, \hat{v}_{i}, s_{i}\right)\right)-T_{i}\left(\hat{v}_{i}, \sigma_{i}\left(v_{i}, \hat{v}_{i}, s_{i}\right)\right)\right] d G_{i}\left(s_{i}\right) .
$$

Using (15) and Lemma 4 we have,

$$
\pi_{i}\left(v_{i}, \hat{v}_{i}\right)=\int \tilde{\pi}_{i}\left(\sigma_{i}\left(v_{i}, \hat{v}_{i}, s_{i}\right), \sigma_{i}\left(v_{i}, \hat{v}_{i}, s_{i}\right) ; \hat{v}_{i}\right) d G_{i}\left(s_{i}\right)
$$

Suppose $v_{i}>\hat{v}_{i}$. Note that by (15) and the monotonicity of $u_{i}$, we have $\sigma_{i}\left(v_{i}, \hat{v}_{i}, s_{i}\right)>$ $s_{i}>\sigma_{i}\left(\hat{v}_{i}, v_{i}, s_{i}\right)$. By $(14)$, we can rewrite (40) as

$$
\pi_{i}\left(v_{i}, \hat{v}_{i}\right)=\int\left[\tilde{\pi}_{i}\left(s_{i}, s_{i} ; \hat{v}_{i}\right)+\int_{s_{i}}^{\sigma_{i}\left(v_{i}, \hat{v}_{i}, s_{i}\right)} u_{i 2}\left(\hat{v}_{i}, z\right) X_{i}\left(\hat{v}_{i}, z\right) d z\right] d G_{i}\left(s_{i}\right) .
$$


This becomes, by $\pi_{i}\left(\hat{v}_{i}, \hat{v}_{i}\right)=\int \tilde{\pi}_{i}\left(s_{i}, s_{i}, \hat{v}_{i}\right) d G_{i}\left(s_{i}\right)$,

$$
\pi_{i}\left(v_{i}, \hat{v}_{i}\right)=\pi_{i}\left(\hat{v}_{i}, \hat{v}_{i}\right)+\iint_{s_{i}}^{\sigma_{i}\left(v_{i}, \hat{v}_{i}, s_{i}\right)} u_{i 2}\left(\hat{v}_{i}, z\right) X_{i}\left(\hat{v}_{i}, z\right) d z d G_{i}\left(s_{i}\right)
$$

Note that $\sigma_{i}$ defined by (15) is continuous and monotonic. Hence the image of $\sigma_{i}\left(y, \hat{v}_{i}, s_{i}\right)$ on $y \in\left[\hat{v}_{i}, v_{i}\right]$ is $\left[s_{i}, \sigma_{i}\left(v_{i}, \hat{v}_{i}, s_{i}\right)\right]$, and thus we can change the variable of the inside integral in (41) from $z \in\left[s_{i}, \sigma_{i}\left(v_{i}, \hat{v}_{i}, s_{i}\right)\right]$ to $y \in\left[\hat{v}_{i}, v_{i}\right]$ to get

$\pi_{i}\left(v_{i}, \hat{v}_{i}\right)=\pi_{i}\left(\hat{v}_{i}, \hat{v}_{i}\right)+\iint_{\hat{v}_{i}}^{v_{i}} u_{i 2}\left(\hat{v}_{i}, \sigma_{i}\left(y, \hat{v}_{i}, s_{i}\right)\right) X_{i}\left(\hat{v}_{i}, \sigma_{i}\left(y, \hat{v}_{i}, s_{i}\right)\right) \sigma_{i 1}\left(y, \hat{v}_{i}, s_{i}\right) d y d G_{i}\left(s_{i}\right)$.

By differentiating (15) in $v_{i}$ (using the Implicit Function Theorem),

$$
u_{i 1}\left(v_{i}, s_{i}\right)=u_{i 2}\left(\hat{v}_{i}, \sigma_{i}\left(v_{i}, \hat{v}_{i}, s_{i}\right)\right) \sigma_{i 1}\left(v_{i}, \hat{v}_{i}, s_{i}\right)
$$

The last two equations imply (16) for the case of $v_{i}>\hat{v}_{i}$. An identical argument yields the same for $v_{i}<\hat{v}_{i}$.

Proof of Lemma 6. Using (16), incentive compatibility in the first round (the inequality-system (17)) is equivalent to, for all $v_{i}>\hat{v}_{i}$,

$$
\begin{aligned}
\int \frac{\int_{\hat{v}_{i}}^{v_{i}} u_{i 1}\left(y, s_{i}\right) X_{i}\left(\hat{v}_{i}, \sigma_{i}\left(y, \hat{v}_{i}, s_{i}\right)\right) d y}{v_{i}-\hat{v}_{i}} d G_{i}\left(s_{i}\right) \leq \frac{\pi_{i}\left(v_{i}, v_{i}\right)-\pi_{i}\left(\hat{v}_{i}, \hat{v}_{i}\right)}{v_{i}-\hat{v}_{i}} \\
\leq \int \frac{\int_{\hat{v}_{i}}^{v_{i}} u_{i 1}\left(y, s_{i}\right) X_{i}\left(v_{i}, \sigma_{i}\left(y, v_{i}, s_{i}\right)\right) d y}{v_{i}-\hat{v}_{i}} d G_{i}\left(s_{i}\right) .
\end{aligned}
$$

By $X_{i} \leq 1$ and the concavity of $u_{i}$ in $v_{i}$,

$$
\frac{\int_{\hat{v}_{i}}^{v_{i}} u_{i 1}\left(y, s_{i}\right) X_{i}\left(\hat{v}_{i}, \sigma_{i}\left(y, \hat{v}_{i}, s_{i}\right)\right) d y}{v_{i}-\hat{v}_{i}} \leq \frac{\int_{\hat{v}_{i}}^{v_{i}} u_{i 1}\left(y, s_{i}\right) d y}{v_{i}-\hat{v}_{i}} \leq u_{i 1}\left(\hat{v}_{i}, s_{i}\right),
$$

and by assumption $u_{i 1}\left(\hat{v}_{i}, s_{i}\right)$ has a finite expectation with respect to $s_{i}$. Therefore, by the Lebesgue Convergence Theorem,

$$
\begin{aligned}
& \lim _{v_{i} \rightarrow \hat{v}_{i}} \int \frac{\int_{\hat{v}_{i}}^{v_{i}} u_{i 1}\left(y, s_{i}\right) X_{i}\left(\hat{v}_{i}, \sigma_{i}\left(y, \hat{v}_{i}, s_{i}\right)\right) d y}{v_{i}-\hat{v}_{i}} d G_{i}\left(s_{i}\right)= \\
& \quad \int \lim _{v_{i} \rightarrow \hat{v}_{i}} \frac{\int_{\hat{v}_{i}}^{v_{i}} u_{i 1}\left(y, s_{i}\right) X_{i}\left(\hat{v}_{i}, \sigma_{i}\left(y, \hat{v}_{i}, s_{i}\right)\right) d y}{v_{i}-\hat{v}_{i}} d G_{i}\left(s_{i}\right)=\int u_{i 1}\left(\hat{v}_{i}, s_{i}\right) X_{i}\left(\hat{v}_{i}, s_{i}\right) d G_{i}\left(s_{i}\right) .
\end{aligned}
$$


By analogous reasoning,

$$
\lim _{\hat{v}_{i} \rightarrow v_{i}} \int \frac{\int_{\hat{v}_{i}}^{v_{i}} u_{i 1}\left(y, s_{i}\right) X_{i}\left(v_{i}, \sigma_{i}\left(y, v_{i}, s_{i}\right)\right) d y}{v_{i}-\hat{v}_{i}} d G_{i}\left(s_{i}\right)=\int u_{i 1}\left(v_{i}, s_{i}\right) X_{i}\left(v_{i}, s_{i}\right) d G_{i}\left(s_{i}\right) .
$$

Therefore, if $X_{i}$ is continuous in $v_{i}$ then $\Pi_{i}\left(v_{i}\right) \equiv \pi_{i}\left(v_{i}, v_{i}\right)$ is differentiable everywhere, and

$$
\Pi_{i}^{\prime}\left(v_{i}\right)=\int u_{i 1}\left(v_{i}, s_{i}\right) X_{i}\left(v_{i}, s_{i}\right) d G_{i}\left(s_{i}\right)
$$

Since this derivative is finite for all $v_{i}, \Pi_{i}$ is Lipschitz-continuous and hence it can be recovered from its derivative, and we obtain (18).

Proof of Proposition 2. If, for all $j=1, \ldots, n$ and $v_{j} \in[\underline{v}, \bar{v}]$, type $v_{j}$ of buyer $j$ purchases a premium $p_{j}\left(v_{j}\right)=\left(1-F_{j}\left(v_{j}\right)\right) / f_{j}\left(v_{j}\right) u_{i 1}$ then buyer $i$ will win in the second round if and only if, for all $j$,

$$
u_{i}\left(v_{i}, s_{i}\right)-\frac{1-F_{i}\left(v_{i}\right)}{f_{i}\left(v_{i}\right)} u_{i 1} \geq \max \left\{u_{j}\left(v_{j}, s_{j}\right)-\frac{1-F_{j}\left(v_{j}\right)}{f_{j}\left(v_{j}\right)} u_{i 1}, 0\right\} .
$$

This is so because in the second round, every buyer $j$ bids $u_{j}\left(v_{j}, s_{j}\right)-p_{j}\left(v_{j}\right)$ by the assumption that the buyers follow weakly dominant strategies in the second round. Hence the allocation rule is indeed the same as in the optimal mechanism, provided that all buyers behave "truthfully," i.e., every buyer $j$ with type $v_{j}$ chooses $p_{j}\left(v_{j}\right)$ for a fee $c_{j}\left(v_{j}\right)$ defined in $(24)$.

We show that the handicap auction defined by (23) and (24) is incentive compatible. Let $\pi_{i}^{h}\left(v_{i}, \hat{v}_{i}\right)$ denote the payoff of buyer $i$ with type $v_{i}$ if he "deviates" to $\hat{v}_{i}$ (chooses $c_{i}\left(\hat{v}_{i}\right)$ and $\left.p_{i}\left(\hat{v}_{i}\right)\right)$. First, we show that for buyer $i$ there is no incentive to deviate downwards. Suppose $\hat{v}_{i}<v_{i}$. Then the deviator bids $u_{i}\left(v_{i}, s_{i}\right)-p_{i}\left(\hat{v}_{i}\right)$ in the second round and his payoff is

$$
\pi_{i}^{h}\left(v_{i}, \hat{v}_{i}\right)=-c_{i}\left(\hat{v}_{i}\right)+\iint \max \left\{u_{i}\left(v_{i}, s_{i}\right)-p_{i}\left(\hat{v}_{i}\right)-W_{-i}^{0}\left(v_{-i}, s_{-i}\right), 0\right\} d F_{-i} d G
$$

Using the definitions of $c_{i}$ and $W_{i}$, this can be rewritten as

$$
\begin{aligned}
& \iiint_{\underline{v}}^{\hat{v}_{i}} u_{i 1} \mathbf{1}_{\left\{W_{i}\left(y, s_{i}\right) \geq W_{-i}^{0}\left(v_{-i}, s_{-i}\right)\right\}} d y-\max \left\{u_{i}\left(\hat{v}_{i}, s_{i}\right)-p_{i}\left(\hat{v}_{i}\right)-W_{-i}^{0}\left(v_{-i}, s_{-i}\right), 0\right\} d F_{-i} d G \\
& -\iint \max \left\{u_{i}\left(v_{i}, s_{i}\right)-p_{i}\left(\hat{v}_{i}\right)-W_{-i}^{0}\left(v_{-i}, s_{-i}\right), 0\right\} d F_{-i} d G .
\end{aligned}
$$


Notice, if $v_{i}=\hat{v}_{i}$, then the last two terms cancel each others, therefore the integral in the first line equals $\pi_{i}^{h}\left(\hat{v}_{i}, \hat{v}_{i}\right)$. This implies that

$$
\pi_{i}^{h}\left(v_{i}, v_{i}\right)=\pi_{i}^{h}\left(\hat{v}_{i}, \hat{v}_{i}\right)+\iint\left[\int_{\hat{v}_{i}}^{v_{i}} u_{i 1} \mathbf{1}_{\left\{W_{i}\left(y, s_{i}\right) \geq W_{-i}^{0}\left(v_{-i}, s_{-i}\right)\right\}} d y\right] d F_{-i} d G
$$

and

$$
\begin{aligned}
\pi_{i}^{h}\left(v_{i}, \hat{v}_{i}\right) & =\pi_{i}^{h}\left(\hat{v}_{i}, \hat{v}_{i}\right)-\iint \max \left\{u_{i}\left(\hat{v}_{i}, s_{i}\right)-p_{i}\left(\hat{v}_{i}\right)-W_{-i}^{0}\left(v_{-i}, s_{-i}\right), 0\right\} d F_{-i} d G \\
& +\iint \max \left\{u_{i}\left(v_{i}, s_{i}\right)-p_{i}\left(\hat{v}_{i}\right)-W_{-i}^{0}\left(v_{-i}, s_{-i}\right), 0\right\} d F_{-i} d G
\end{aligned}
$$

Since $\partial u_{i}\left(v_{i}, s_{i}\right) / \partial v_{i}=u_{i 1}$ is a constant, $u_{i}\left(v_{i}, s_{i}\right)$ can be written as $u_{i 1} v_{i}+r_{i}\left(s_{i}\right)$. We claim that the difference between the integrands in the second and third terms can be rewritten as,

$$
\begin{aligned}
& \max \left\{u_{i 1} v_{i}+r_{i}\left(s_{i}\right)-p_{i}\left(\hat{v}_{i}\right)-W_{-i}^{0}\left(v_{-i}, s_{-i}\right), 0\right\}-\max \left\{u_{i 1} \hat{v}_{i}+r_{i}\left(s_{i}\right)-p_{i}\left(\hat{v}_{i}\right)-W_{-i}^{0}\left(v_{-i}, s_{-i}\right), 0\right\} \\
& =\int_{\hat{v}_{i}}^{v_{i}} u_{i 1} \mathbf{1}_{\left\{u_{i 1} y+r_{i}\left(s_{i}\right)-p_{i}\left(\hat{v}_{i}\right) \geq W_{-i}^{0}\left(v_{-i}, s_{-i}\right)\right\}} d y .
\end{aligned}
$$

If $u_{i 1} \hat{v}_{i}+r_{i}\left(s_{i}\right)-p_{i}\left(\hat{v}_{i}\right) \geq W_{-i}^{0}\left(v_{-i}, s_{-i}\right)$ then the difference is

$$
u_{i 1}\left(v_{i}-\hat{v}_{i}\right)=\int_{\hat{v}_{i}}^{v_{i}} u_{i 1} d y=\int_{\hat{v}_{i}}^{v_{i}} u_{i 1} \mathbf{1}_{\left\{u_{i 1} y+r_{i}\left(s_{i}\right)-p_{i}\left(\hat{v}_{i}\right) \geq W_{-i}^{0}\left(v_{-i}, s_{-i}\right)\right\}} d y
$$

Suppose $u_{i 1} v_{i}+r_{i}\left(s_{i}\right)-p_{i}\left(\hat{v}_{i}\right) \geq W_{-i}^{0}\left(v_{-i}, s_{-i}\right) \geq u_{i 1} \hat{v}_{i}+r_{i}\left(s_{i}\right)-p_{i}\left(\hat{v}_{i}\right)$. Let $y^{*} \in\left\{\hat{v}_{i}, v_{i}\right\}$ be such that $W_{-i}^{0}\left(v_{-i}, s_{-i}\right)=u_{i 1} v_{i}+r_{i}\left(s_{i}\right)-p_{i}\left(\hat{v}_{i}\right)$. Then (45) is

$$
\begin{aligned}
u_{i 1} v_{i}+r_{i}\left(s_{i}\right)-p_{i}\left(\hat{v}_{i}\right)-W_{-i}^{0}\left(v_{-i}, s_{-i}\right) & =u_{i 1}\left(v_{i}-y^{*}\right)=\int_{y^{*}}^{v_{i}} u_{i 1} d y \\
& =\int_{\hat{v}_{i}}^{v_{i}} u_{i 1} \mathbf{1}_{\left\{u_{i 1} y+r_{i}\left(s_{i}\right)-p_{i}\left(\hat{v}_{i}\right) \geq W_{-i}^{0}\left(v_{-i}, s_{-i}\right)\right\}} d y .
\end{aligned}
$$

If $W_{-i}^{0}\left(v_{-i}, s_{-i}\right) \geq u_{i 1} y+r_{i}\left(s_{i}\right)-p_{i}\left(\hat{v}_{i}\right)$ then the value of both sides of (45) is zero. Therefore (45) indeed holds. From (44) and (45) it follows that

$$
\pi_{i}^{h}\left(v_{i}, \hat{v}_{i}\right)=\pi_{i}^{h}\left(\hat{v}_{i}, \hat{v}_{i}\right)+\iint\left[\int_{\hat{v}_{i}}^{v_{i}} u_{i 1} \mathbf{1}_{\left\{u_{i 1} y+r_{i}\left(s_{i}\right)-p_{i}\left(\hat{v}_{i}\right) \geq W_{-i}^{0}\left(v_{-i}, s_{-i}\right)\right\}} d y\right] d F_{-i} d G
$$


Notice that since $p_{i}$ is decreasing, $u_{i 1} y+r_{i}\left(s_{i}\right)-p_{i}\left(\hat{v}_{i}\right) \leq u_{i 1} y+r_{i}\left(s_{i}\right)-p_{i}(y)$ whenever $y \in\left\{\hat{v}_{i}, v_{i}\right\}$. Hence

$\pi_{i}^{h}\left(v_{i}, \hat{v}_{i}\right) \leq \pi_{i}^{h}\left(\hat{v}_{i}, \hat{v}_{i}\right)+\iint\left[\int_{\hat{v}_{i}}^{v_{i}} u_{i 1} \mathbf{1}_{\left\{u_{i 1} y+r_{i}\left(s_{i}\right)-p_{i}(y) \geq W_{-i}^{0}\left(v_{-i}, s_{-i}\right)\right\}} d y\right] d F_{-i} d G=\pi_{i}^{h}\left(v_{i}, v_{i}\right)$

where the equality follows from (43). This means that for buyer $i$ there is no incentive to deviate downwards. An almost identical argument shows that there is no incentive to deviate upwards either.

\section{References}

[1] Baron, D, and D. Besanko (1984), "Regulation and Information in a Continuing Relationship," Information Economics and Policy, 1:267-302.

[2] Bergemann, D., and M. Pesendorfer (2002), "Information Structures in Optimal Auctions," Cowles Foundation Discussion Paper No. 1323

[3] Bulow, J., and J. Roberts (1989), "The Simple Economics of Optimal Auctions," Journal of Political Economy, 97:1060-1090.

[4] Compte, O., and P. Jehiel (2001), "Auctions and Information Acquisition: Sealedbid or Dynamic Formats?," mimeo, C.E.R.A.S.

[5] Courty, P., and Li (2000), "Sequential Screening," Review of Economic Studies, 67:697-717.

[6] Crémer, J., and R. P. McLean (1988), "Full Extraction of the Surplus in Bayesian and Dominant Strategy Auctions," Econometrica, 53:345-361.

[7] Dasgupta, P., and E. Maskin (2000), "Efficient Auctions," The Quarterly Journal of Economics, 115(2):341-388.

[8] Demsetz, H. (1968), "Why Regulate Utilities?" Journal of Law and Economics, 11:55-65.

[9] French, K., and R. McCormick (1984), "Sealed Bids, Sunk Costs, and the Process of Competition," Journal of Business, 57:417-441.

[10] Ganuza, J-J, (2003), "Ignorance Promotes Competition: An Auction Model with Endogenous Private Valuations," forthcoming, RAND Journal of Economics.

[11] Gershkov (2002), "Optimal Auctions and Endogenous Information Structure," mimeo. 
[12] Harris, M., and A. Raviv (1981), "Allocations Mechanisms and the Design of Auctions," Econometrica 49:1477-1499.

[13] Krigman, L., W.H. Shaw, and K.L. Womack (2001), "Why Do Firms Switch Underwriters?" Journal of Financial Economics 60:245-284.

[14] Levin, D., and J. Smith (1994), "Equilibrium in Auctions with Entry," American Economic Review, 84:585-599.

[15] Loeb, M., and W. A. Magat (1979), "A Decentralized Method of Utility Regulation," Journal of Law and Economics, 22:399-404.

[16] Maskin, E., and J. Riley (1984), "Monopoly with Incomplete Information," RAND Journal of Economics, 15:171-96.

[17] McAfee, P. R, and J. McMillan (1987), "Auctions with Entry," Economics Letters, 23:343-347.

[18] Milgrom, P., and R. Weber (1982), "A Theory of Auctions and Competitive Bidding," Econometrica, 50:1089-1122.

[19] Moel, A., and P. Tufano (1997), "Bidding for Antamina," HBS case \#9-297-054.

[20] Myerson, R. (1981), "Optimal Auction Design," Mathematics of Operations Research 6:58-73.

[21] Persico, N. (2000), "Information Acquisition in Auctions," Econometrica, 68:135148.

[22] Riley, J., and W. Samuelson (1981), "Optimal Auctions," American Economic Review, 71:381-92.

[23] Riordan, M., and D. Sappington (1987), "Awarding Monopoly Franchises," American Economic Review, 77:375-387.

[24] Royden, H. L. (1967), Real Analysis, The McMillan Company, New York. 